\title{
Approximate Techniques for Predicting Size Effects on Cleavage Fracture Toughness $(J c)$
}

Manuscript Completed: February 1993

Date Published: July 1993

Prepared by

M. T. Kirk, R. H. Dodds, Jr., University of Illinois

University of Illinois at Urbana-Champaign

Department of Civil Engineering, MC-250

205 North Mathews Avenue

Urbana, Il 61801-2352

Under Contract to:

Naval Surface Warfare Center

Annapolis Detachment, Carderock Division

Code 2814

Annapolis, MD 21402-5067

\section{Prepared for}

Division of Engineering

Office of Nuclear Regulatory Research

U.S. Nuclear Regulatory Commission

Washington, DC 20555-0001

NRC FIN B6290

\section{MASIER}




\begin{abstract}
This investigation examines the ability of an elastic $T$-stress analysis coupled with a modified boundary layer $(M B L)$ solution to predict stresses ahead of a crack tip in a variety of planar geometries. The approximate stresses are used as input to estimate the effective driving force for cleavage fracture $\left(J_{0}\right)$ using the micromechanically based approached introduced by Dodds and Anderson. Finite element analyses for a wide variety of planar cracked geometries are conducted which have elastic biaxiality parameters $(\beta)$ ranging from -0.99 (very low constraint) to +2.96 (very high constraint). The magnitude and sign of $\beta$ indicate the rate at which crack-tip constraint changes with increasing applied load. All results pertain to a moderately strain hardening hardening material (strain hardening exponent $(n)$ of 10 ). These analyses suggest that $\beta$ is an effective indicator of both the accuracy of $T-M B L$ estimates of $J_{o}$ and of applicability limits on evolving fracture analysis methodologies (i.e. $T-M B L, J-Q$, and $J / J_{o}$ ). Specifically, when $|\beta|>0.4$ these analyses show that the $T-M B L$ approximation of $J_{0}$ is accurate to within $20 \%$ of a detailed finite-element analysis. As "structural type" configurations, i.e. shallow cracks in tension, generally have $|\beta|>0.4$, it appears that only an elastic analysis may be needed to determine reasonably accurate $J_{o}$ values for structural conditions.
\end{abstract}

Keywords: Elastic-plastic fracture mechanics, size effects, finite element analysis, modified boundary layer, $T$-stress, $J_{S S Y}, J_{o}, Q$, structural integrity assessment. 


\section{Contents}

Section No.

Page

Abstract

iii

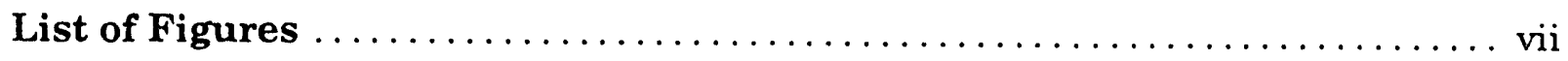

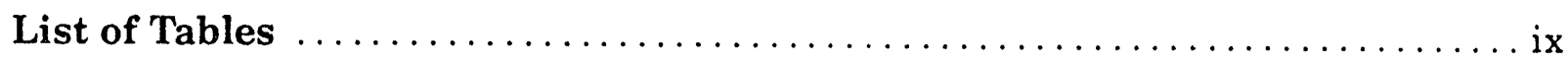

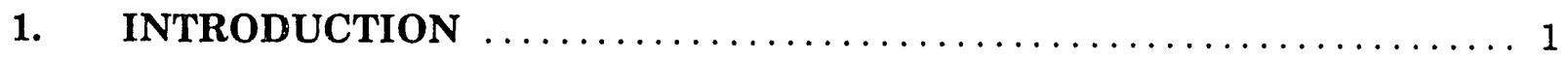

1.1 Crack-Tip Stress Fields $\ldots \ldots \ldots \ldots \ldots \ldots \ldots \ldots \ldots \ldots \ldots \ldots \ldots$

$1.2 \mathrm{~T}$ and $\mathrm{Q}$ as Constraint Parameters $\ldots \ldots \ldots \ldots \ldots \ldots \ldots \ldots \ldots$

1.3 Micromechanical Models for Cleavage Fracture $\ldots \ldots \ldots \ldots \ldots \ldots . \ldots$

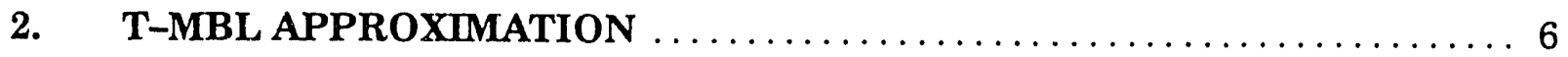

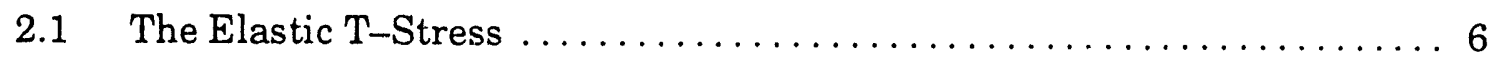

2.2 Modified Boundary Layer Model $\ldots \ldots \ldots \ldots \ldots \ldots \ldots \ldots \ldots \ldots$

2.3 Approximation of Opening Mode Stresses . ..................6 6

3. MICROMECHANICS PREDICTIONS OF SIZE EFFECTS ON $J_{c} \ldots \ldots .8$

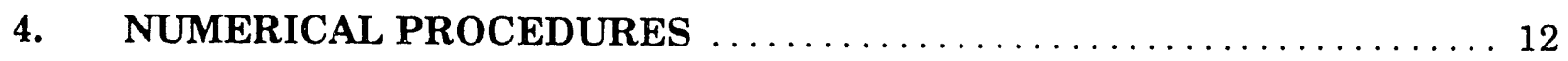

4.1 Constitutive Model ................................ 12

$4.2 \quad$ Finite Elements and Crack-Tip Modelling ................... 13

4.3 Modified Boundary Layer (MBL) Model $\ldots \ldots \ldots \ldots \ldots \ldots \ldots \ldots 13$

4.4 Models of Finite Geometries ............................ 15

4.5 Post Processing to Obtain $J$, CTOD, $J_{0}$, and $\beta \ldots \ldots \ldots \ldots \ldots \ldots \ldots$

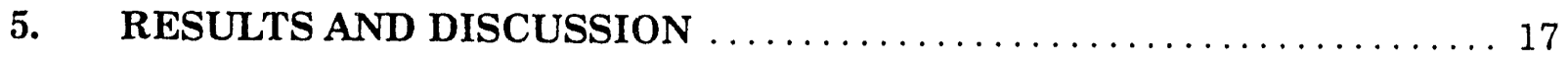

5.1 Modified Boundary Layer Solutions $\ldots \ldots \ldots \ldots \ldots \ldots \ldots \ldots \ldots \ldots$

5.2 Opening Mode Stresses in Finite Bodies $\ldots \ldots \ldots \ldots \ldots \ldots \ldots \ldots \ldots \ldots$

5.3 $J_{o}$ Estimation Deformation Limits on the Applicability of these Fracture Analysis Methodologies ........................... 18

5.4 Deformation Limits on the Applicability of these Fracture Analysis Methodologies 


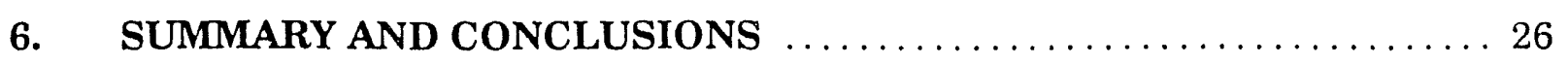

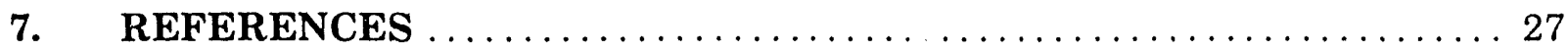




\section{List of Figures}

Figure No.

Page

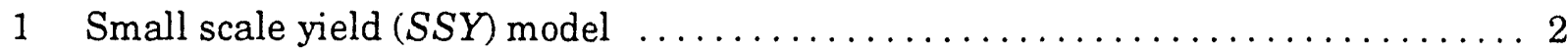

2 Effect of infinite body reference solution on radial independence of $Q$ for an $a / W=0.15 \mathrm{SE}(\mathrm{B})$ specimen having a Ramberg-Osgood strain hardening exponent

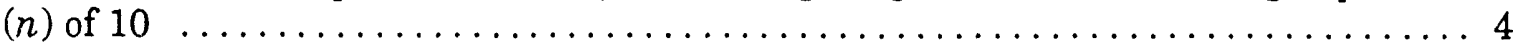

3 Schematic illustration of fracture toughness locus constructed in mid-transition using various planar specimen geometries to obtain $J_{\text {crit }}$ over a range of constraint

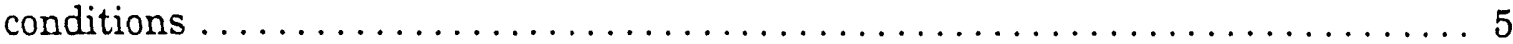

4 Relationship between opening mode stress and elastic $T$-stress quantified by a modified boundary layer model. Similar curves can be constructed at any constant normalized distance, $\Re_{R}$, ahead of the crack tip from finite

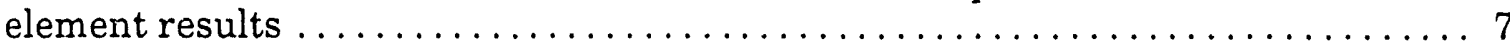

5 Conceptual variation of $J_{0}$ with $J$ for two finite bodies. Horizontal lines $\left(J_{0}=\right.$ constant $)$ denote equivalent driving force for cleavage fracture (equivalent stressed volumes) in both bodies

6 Effect of applied load on opening mode stresses ahead of a crack in a finite body, after [Kirk and Dodds, 1992] . .......................... 10

7 Opening mode stresses on the crack plane in SSY for Ramberg-Osgood materials [Kirk and Dodds, 1992] ............................ 11

8 Effect of stress errors in estimated opening mode stress on predicted toughness values. These results depend on $\sigma_{f}^{*}$, however the relative effect of strain hardening $(n)$ is independent of $\sigma_{f}^{*} \ldots \ldots \ldots \ldots \ldots \ldots \ldots \ldots \ldots$. 11

9 Uniaxial stress-strain curve defined by eon. (10) $\ldots \ldots \ldots \ldots \ldots \ldots \ldots \ldots$

10 Finite element mesh for double cantilever beam specimen $\ldots \ldots \ldots \ldots \ldots \ldots 14$

11 Opening mode stresses on the crack plane from modified boundary

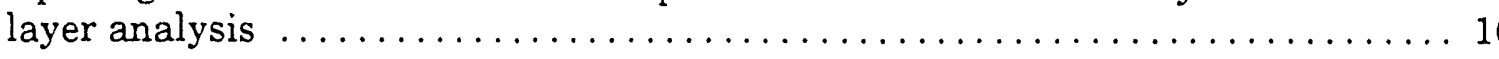

12 Variation of opening mode stress on the crack plane with applied $T$-stress and distance ahead of the crack tip determined by modified boundary layer analysis

13 Comparison of modified boundary layer approximation with opening mode tresses in various planar cracked geometries for an $n=10$, $\sigma_{o} / E=0.002$ material .....................................

14 Comparison of modified boundary layer approximation with opening mode stresses in various planar cracked geometries grouped by $|\beta|$ values for an $n=10$,

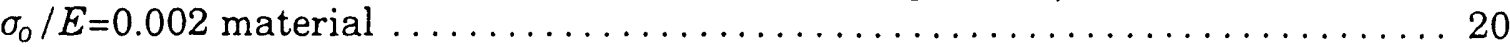

15 Comparison of constraint correction curves for cleavage fracture determined by elastic-plastic finite element analysis with those determined by the $T-M B L$ approximation for an $n=10, \sigma_{o} / E=0.002$ material 
16 Effect of modified boundary layer stress estimation accuracy on $J_{o}$ estimation

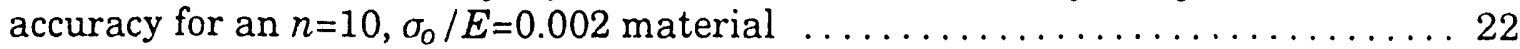

17 Postulated effect of material strain hardening on the range of biaxiality parameter $(\beta)$ over which $T-M B L J_{o}$ estimates have acceptable accuracy

18 Deformation limits on the $T-M B L$ approximation for $J_{o}$ (top graph) and on elastic-plastic finite element calculation of $J_{0}$ (bottom graph) for an $n=10, \sigma_{0} / E=0.002$ material. Symbols representing specimens of same type are connected by lines. Symbols on top graph marked with asterisks denote deformation when $T / \sigma_{o}=0.8$. At this deformation the $T-M B L$ approximation has the error given in parenthesis 


\section{List of Tables}

Table No.

Page

1. $\beta$ values for planar cracked geometries modelled $\ldots \ldots \ldots \ldots \ldots \ldots \ldots \ldots$ 


\section{Approximate Techniques for Predicting Size Effects on Cleavage Fracture Toughness $\left(J_{c}\right)$}

\section{INTRODUCTION}

Numerous experimental studies report an increase of the cleavage fracture toughness, $J_{c}$, for steels (e.g. A36, A515, HY-80, A533B) as the crack depth is reduced in SE(B) specimens [Sorem, et al., 1991; Kirk, et al., 1991; Sumpter and Forbes, 1992; Theiss and Bryson, 1991]. Cleavage fracture toughness also increases with the transition from bending to tensile load [Keeney-Walker, et al., 1992]. These increases of $J_{c}$ develop when the in-plane plastic flow produced by gross deformation of the specimen impinges on the local crack-tip fields. This relaxes the kinematic constraint against further plastic flow. Once the global and local plastic fields interact, the crack-tip stresses and strains no longer increase in proportion to one another with amplitude governed by $J$ alone. At these high deformation levels, equivalence of $J$ (or CTOD) between different cracked geometries does not insure identical crack-tip stress and strain fields. Because the micro-mechanisms of fracture require attainment of critical conditions described in terms of stress and/or strain, different values of applied $J$ may be required to cause fracture in different structures. These interrelated effects of geometry and loading mode on near-tip stresses, near-tip strains, and fracture toughness are referred to collectively as "size effects." The routinely observed size effects on $J_{c}$ are actually a size effect on the relation between macroscopic fracture parameters (e.g. $J$ ) and micro-scale crack driving force (e.g. opening mode stress). These size effects can be quantified for cleavage fracture by coupling a description of the stress field surrounding the crack tip with appropriate micromechanical failure models. The following sections discuss recent developments in these areas.

\subsection{Crack-Tip Stress Fields}

Small scale yielding ( $S S Y$ ) conditions exist when the crack-tip plastic zone is infinitesimally small compared to all other characteristic lengths and is embedded within a linear-elastic field. Elastic-plastic crack-tip fields in SSY are generated by applying displacements consistent with the first two terms of the linear elastic crack-tip fields to a circular region containing an edge crack (Figure 1):

$$
\begin{aligned}
& \mathrm{u}(r, \theta)=K_{I} \frac{1-\nu}{E} \sqrt{\frac{r}{2 \pi}} \cos \left(\frac{\theta}{2}\right)(3-4 \nu-\cos \theta)+T \frac{1-\nu^{2}}{E} r \cos \theta \\
& \mathrm{v}(r, \theta)=K_{I} \frac{1-\nu}{E} \sqrt{\frac{r}{2 \pi}} \sin \left(\frac{\theta}{2}\right)(3-4 \nu-\cos \theta)-T \frac{\nu(1+\nu)}{E} r \sin \theta
\end{aligned}
$$

$K_{I}$ and $T$ are the leading coefficients of an asymptotic expansion for stresses surrounding the crack tip in a two dimensional linear-elastic body:

$$
\begin{aligned}
& \sigma_{x}=\frac{K_{I}}{\sqrt{2 \pi r}} f_{x}(\theta)+T+O(\sqrt{r}) \\
& \sigma_{y}=\frac{K_{I}}{\sqrt{2 \pi r}} f_{y}(\theta)+O(\sqrt{r}) \\
& \tau_{x y}=\frac{K_{I}}{\sqrt{2 \pi r}} f_{x y}(\theta)+O(\sqrt{r})
\end{aligned}
$$




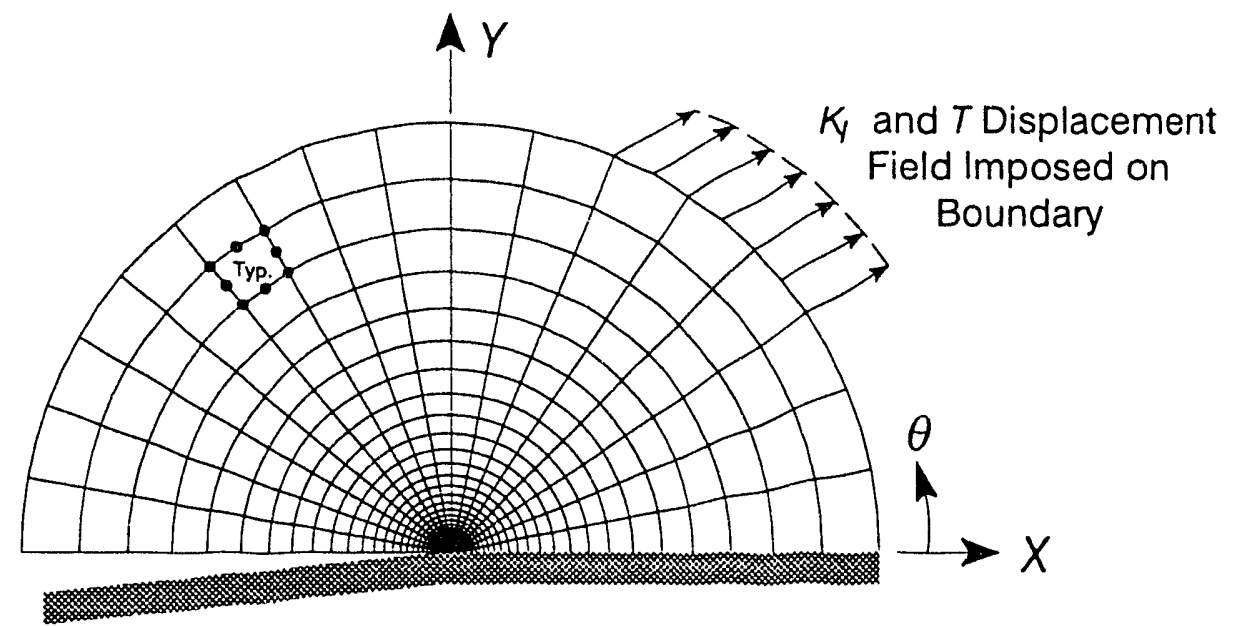

Figure 1: Small scale yield (SSY) model.

Here $r$ is the radial distance from the crack tip, $K_{I}$ is the stress intensity factor, and $T$ is the stress parallel to the crack over vanishingly small $r$. All remaining terms are defined in Figure 1. The truncated terms in eqn. (2) are of order $\sqrt{r}$ or higher and consequently approach zero as $r \rightarrow 0$. Thus, $K$ and $T$ alone control the stress state in the near vicinity of the crack tip under SSY conditions.

Rice and Tracy [1968] and McMeeking [1977] originally proposed the model depicted in Figure 1, with $T=0$, as a boundary layer solution of the infinite body, single-ended crack problem. For this special case, the single parameter $J$ both sets the size scale over which large deformations develop and describes the magnitude of stresses near the crack tip but outside of the finite deformation zone. The HRR field equations quantify the relation between $J$ and crack-tip stresses and strains in an infinite body made of incompressible, fully plastic powerlaw hardening material [Hutchinson, 1968; Rice and Rosengren, 1968].

Larsson and Carlsson [1973] modified the boundary layer ( $M B L)$ solution by applying non-zero $T$ stresses to approximate the effects of finite size on crack-tip region deformation and plastic flow. The $T=0$ (infinite body) solution is one of a family of SSY solutions generated by changing the magnitude of the $T$-stress applied to the $M B L$ model. Larsson and Carlsson found a marked influence of the the sign and magnitude of the $T$-stress on the size and shape of the crack-tip plastic zone. More recent investigations by both Hancock and Parks (and coworkers) [Al-Ani and Hancock, 1991; Bertegon and Hancock, 1991; Sumpter and Hancock, 1991; Parks, 1991; Du and Hancock, 1991; Wang, 1991(a); Wang, 1991(b)] demonstrate a strong influence of $T$-stress on opening mode stresses near the crack tip. Positive $T$ stresses slightly elevate the opening mode stresses relative to the $T=0$ condition while negative $T$ stresses reduce significantly the opening mode stress. Despite this influence on near-tip stresses, the $T$-stress has no effect on $J$ because $T$ is non-singular. These findings demonstrate the inadequacy of $J$ as the sole descriptor of deformation near a crack tip in finite geometries, which generally have non-zero $T$. 
O'Dowd and Shih [1991, 1992] propose a two-parameter theory which describes crack-tip fields in finite geometries $(T \neq 0$ ). They describe stresses surrounding a crack tip in terms of $J$ plus an additional parameter $Q$ :

$$
\frac{\sigma_{i j}}{\sigma_{o}}=\left(\frac{J}{a \varepsilon_{o} \sigma_{o} I_{n} r}\right)^{\frac{1}{n+1}} \tilde{\sigma}_{i j}(\theta ; n)+Q\left(\frac{r}{J / \sigma_{o}}\right)^{q} \hat{\sigma}_{i j}(\theta ; n)
$$

Here, $J$ sets the size scale over which large deformations develop while $Q$ quantifies the opening stress magnitude. O'Dowd and Shih found by analysis of the $M B L$ model that (1) the power on the radial coefficient in the second term $(q)$ is approximately zero for $n \geq 4,(2)$ for $|\theta| \leq 90^{\circ}$ the second-order normal stresses $\left(\hat{\sigma}_{r r}\right.$ and $\hat{\sigma}_{\theta \theta}$ ) are approximately equal, and (3) the second-order shear stress $\left(\hat{\sigma}_{r \theta}\right)$ is approximately zero. Thus, $Q$ is the amplitude of a hydrostatic, or triaxiality term. Based on these observations, eqn. (3) simplifies to:

$$
\frac{\sigma_{i j}}{\sigma_{o}}=\left(\frac{J}{\alpha \varepsilon_{o} \sigma_{o} I_{n} r}\right)^{\frac{1}{n+1}} \tilde{\sigma}_{i j}(\theta ; n)+Q \delta_{i j} \text { for }|\theta| \leq 90^{\circ}
$$

Equation (4) expresses normal stresses in finite bodies as the infinite body HRR solution [Hutchinson, 1968; Rice and Rosengren, 1968] plus an additive constant $(Q)$ which is independent of radial distance from the crack tip. Numerical studies reveal that this model is adequate for deformation up to some geometry dependent limit, beyond which $Q$ becomes radially dependent. Dependence of $Q$ on distance from the crack tip introduces ambiguity into the selection of a $Q$ value to parameterize constraint effects on fracture toughness. Selection of an arbitrary location at which to calculate $Q$ implies knowledge of the critical distance for cleavage fracture which a pure mechanics approach, such as the $J-Q$ theory, seeks to avoid. However, use of an alternative infinite body reference solution improves the radial independence of $Q$ and, thereby, the robustness of the $J-Q$ theory. Figure 2 shows that $Q$ values referenced to a full-field infinite body solution (i.e. $S S Y T=0$ ) remain radially independent to much larger deformations than those referenced to an infinite body solution which includes only the singular term (i.e. HRR). Equation (4) is altered to reflect this change of reference solution:

$$
\sigma_{i j}=\sigma_{i j} I_{\mathrm{SSY} ; \mathrm{T}=0}+Q \sigma_{o} \delta_{i j}
$$

Under $S S Y$ conditions, $T$ relates uniquely to $Q$. Analysis of cracks in finite bodies [O'Dowd and Shih, 1992] reveals that eqn. (5) accurately describes the departure of stresses near the crack tip from the SSY family of solutions well into large scale yielding ( LSY). Thus, $Q$ remains a descriptor of crack-tip stresses at deformation levels beyond those for which $T$ can be defined. However, Figure 2 demonstrates that the crack-tip fields in finite bodies lose self-similarity with the SSY T=0 reference solution at high load levels. Once self-similarity with the infinite body reference solution is lost, the conditions for fracture in a finite body depend on characteristics unique to that body. These conditions cannot be parameterized currently within the context of any two-parameter theory. 

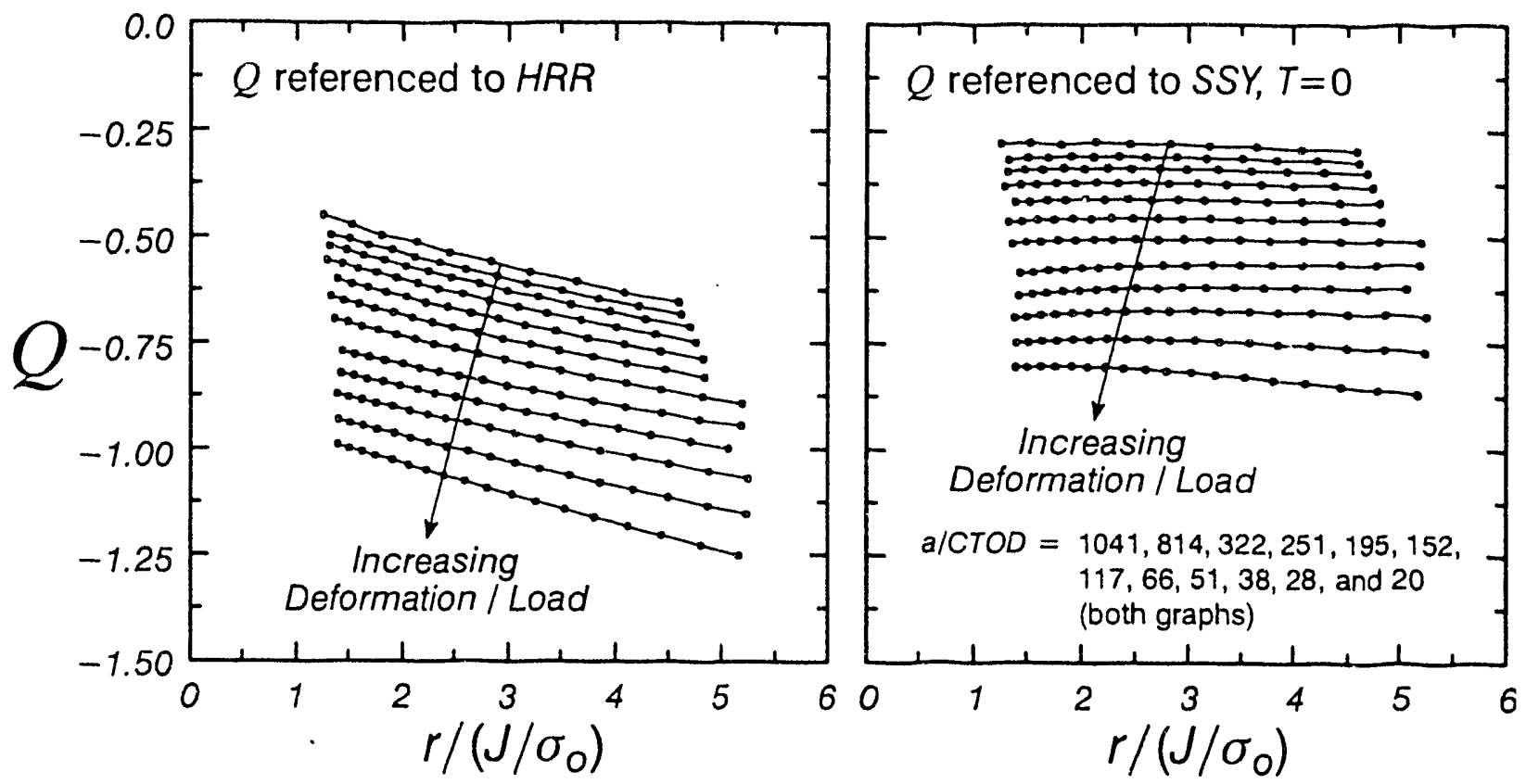

Figure 2: Effect of infinite body reference solution on radial independence of $Q$ for an $\alpha / W=0.15$ $\mathrm{SE}(\mathrm{B})$ specimen having a Ramberg-Osgood strain hardening exponent $(n)$ of 10.

\section{2 $\mathrm{T}$ and $\mathrm{Q}$ as Constraint Parameters}

The linkage of $T$ (in $S S Y$ ) and of $Q$ (in $S S Y$ and $L S Y$ ) with crack-tip stress state motivates the use of $Q$ and $T$ to parameterize constraint effects on fracture toughness data. Several investigators have generated fracture toughness loci [Sumpter and Hancock, 1991; Kirk, et al., 1991; Sumpter and Forbes, 1992], $J_{\text {crit }}$ vs. $Q$ or $J_{\text {crit }}$ vs. $T$, as depicted schematically in Figure 3. However, this approach greatly complicates the determination of fracture toughness; it necessitates conducting difficult experiments with low constraint geometries (e.g. shallow cracks in bending, cracks in tension) to fully define the fracture toughness locus.

Considerable debate surrounds the application of $T$ as a constraint parameter under LSY conditions rather than $Q$. The $T$ approach has the advantage of simplicity relative to $Q$, requiring only a linear-elastic analysis rather than an elastic-plastic analysis of the cracked structure. Under moderate to large scale yielding, the relations between $T$ and the opening mode stresses become geometry dependent. At these deformation levels, opening mode stresses predicted by $T$ only approximate the full elastic-plastic solution quantified by $Q$. However, several numerical studies show that the $T-M B L$ approximation predicts stresses to within $10 \%$ of finite body calculations even for very large deformations.

\subsection{Micromechanical Models for Cleavage Fracture}

Dodds and Anderson [Dodds, et al., 1991; Anderson and Dodds, 1991, Kirk and Dodds, 1992] combine the computation of stresses near the crack tip with the micromechanical conditions for cleavage fracture (achievement of a critical stress over a critical volume [Ritchie, et al., 1973]) to predict the conditions for cleavage fracture in one gecmetry based on toughness data 


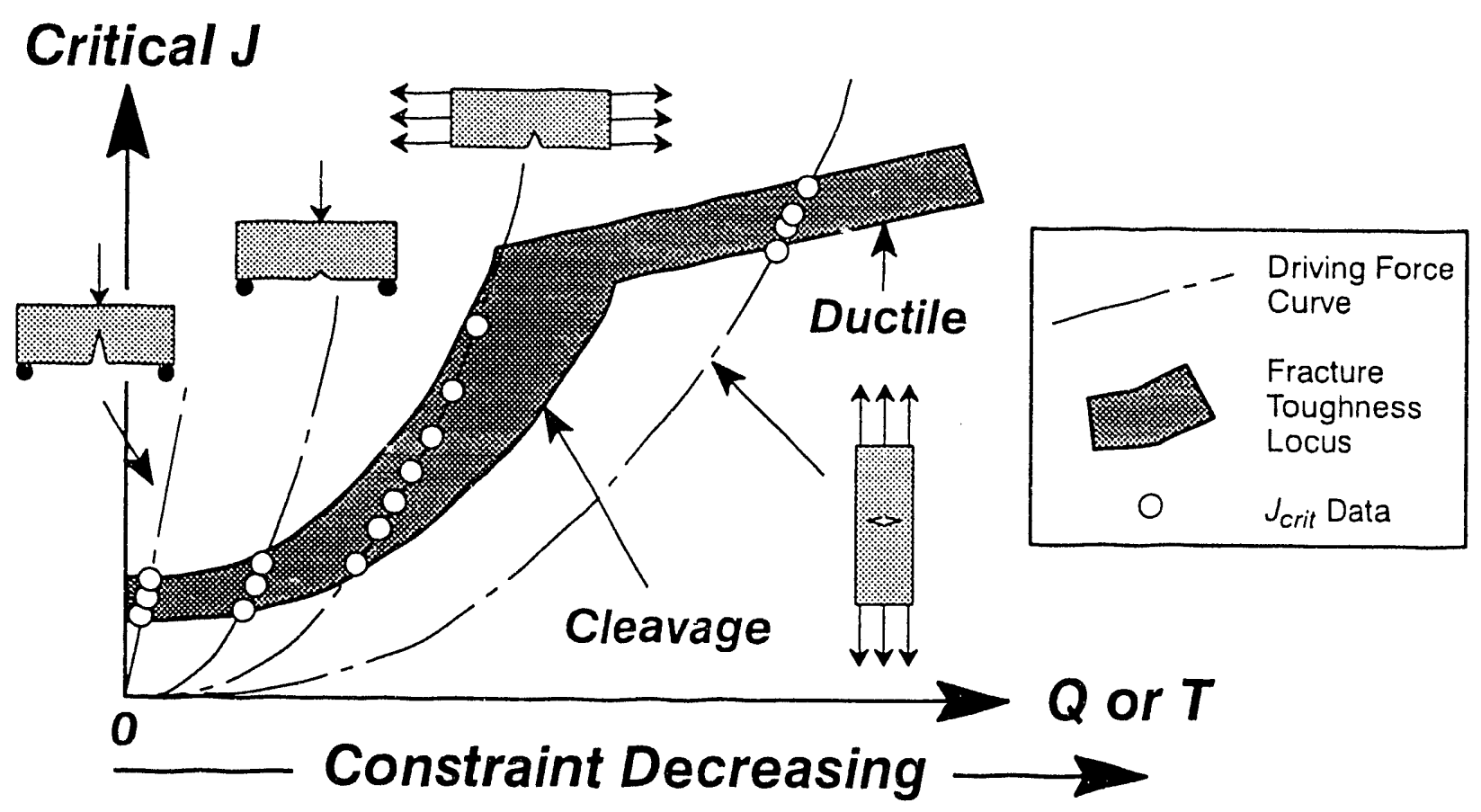

Figure 3: Schematic illustration of fracture tough:ness locus constructed in mid-transition using various planar specimen geometries to obtain $J_{c r i t}$ over a range of constraint conditions.

from another. Experimental data for several steels including mild steel, A36, and A515 in low to mid transition demonstrate the validity of this approach [Sorem, et al., 1991; Kirk, et al., 1991; Sumpter and Forbes, 1992]. For stress controlled cleavage fracture, this micromechani( 1 approach greatly reduces the experimentation needed to define the toughness locus; a considerable simplification over parameterizing constraint effects by either $Q$ or $T$. The micromechanical approach requires detailed resolution of crack-tip fields which, until now, have been obtained by very detailed elastic-plastic finite element analysis. The determination of $Q$ requires computations of equivalent detail. This complication makes such approaches unattractive for routine application to structural fracture safety analysis at this time. In this investigation, the possibility of using the elastic $T$-stress approximations of crack-tip stress distributions as input to the micromechanics analysis is investigated to determine if predictions of size effects having engineering accuracy can be obtained without a detailed elastic-plastic analysis of each configuration considered. 


\section{T-MBL APPROXIMATION}

Two analyses are needed to couple remotely applied load to opening mode stresses near the crack tip:

1. An elastic analysis of the cracked structure that relates applied load to $T$-stress.

2. An elastic-plastic analysis of the $M B L$ model that relates $T$-stress to opening mode stresses near the crack tip.

The synthesis of these two analyses to approximate opening mode stresses in finite bodies is referred to as the $T-M B L$ approximation.

\subsection{The Elastic T-Stress}

The $T$-stress (eqn. (2)) is the non-singular elastic stress acting parallel to the crack over vanishingly small $r$. $T$-stress values for various geornetries are frequently reported in the literature, usually as a non-dimensional ratiu with $K$ :

$$
\beta=\frac{T \sqrt{\pi a}}{K}
$$

where $\beta$ is referred to as a biaxiality parameter which is a constant for a given geometry and loading mode. The various techniques used to compute $T$, and thereby $\beta$, include boundary collocation, second orde weight functions, and domain interaction integrals [Leevers and Radon, 1982; Kfouri, 1986; Sham, 1991; Nakamura and Parks, 1992].

\subsection{Modified Boundary Layer Model}

Details of the $M B L$ model were presented in Section 1.1. Displacements are applied to the $M B L$ model consistent with the first two terms (i.e. $K$ and $T$ ) of the linear solution. Elasticplastic finite element analysis of the model provides a family of full field SSY solutions parametric in $T / \sigma_{0}$. Conditicns are achieved wherein stresses and strains at all angles scale with $r /\left(J /\left(\sigma_{o} \varepsilon_{o} \alpha\right)\right)$, as do the HRR fields. The fields remain self-similar until the plastic zone size becomes a significant portion of the modelled domain radius, $\approx 10 \%$, at which point it senses the finite boundary. Figure 4 schematically depicts the results of such an analysis. The $C_{i}$ coefficients are determined by curve fitting the finite element results at various normalized distances ahead of the crack tip.

\subsection{Approximation of Opening Mode Stresses}

The effect of finite size on opening mode stress near the crack tip is computed by combining the information discussed in the preceding sections. First, an elastic analysis of the cracked geometry is performed to determine $K, T$, and $\beta$. The variation of $T$ with load is determined from eqn. (6) as follows:

$$
\frac{T}{\sigma_{o}}=\frac{\beta K}{\sigma_{o} \sqrt{\pi a}}
$$

These elastic $T$-stress and $K$ values are inserted into the $M B L$ solution (Figure 4) to determine the variation of opening mode stress near the crack tip with applied loading: 


$$
\frac{\sigma_{y y}}{\sigma_{o}}=\sum_{i=0}^{n} C_{i}\left(\frac{\beta K}{\sigma_{o} \sqrt{\pi a}}\right)^{i}
$$

Equation (8) couples the far-field elastic solution with near-tip stresses. The applied load enters this approximation through $K$ while $\beta$ characterizes geometry. Moreover, $\beta$ controls the direction and rate at which increasing load causes deviation of near-tip stresses from the $T=0$ limit. Three examples indicate potential limitations on the applicability of this approximation.

1. $\beta>0$ (high constraint): Equation (8) predicts a continuous increase of normalized opening mode stress with increasing load. This can lead to unrealistic predictions - consider the rectangular double cantilever beam specimen $(a / W=0.5$, $H / W=2.5, \beta=2.96$ [Leevers and Radon, 1982]). At some load the arms of this specimen must form plastic hinges which reduce opening mode stress significantly below the $T-M B L$ prediction.

2. $\beta=0(T \equiv 0)$ : Equation (8) predicts that normalized opening mode stresses remain constant independent of the applied load, i.e. the constraint remains constant at the $T=0$ level. Consider a SE(B) with $a / W=0.39$ which has $\beta=0$ [Leevers and Radon, 1982]. A plastic hinge must form at some load. After hinge formation, plastic flow is no longer contained and opening mode stresses must fall as a consequence.

3. $\beta<0$ (low constraint): Equation (8) predicts the continuous decrease of normalized opening mode stress with increasing load. This behavior is observed in analyses of finite bodies. Two-dimensional cracked geometries having $\beta<0$ include tension loaded central cracks, tension loaded edge cracks $(a / W<0.60)$, edge cracks loaded in bending $(a / W<0.39)$, and double edge cracks. This class of problems also includes the particularly important case of semi-elliptical surface cracks loaded in tension.

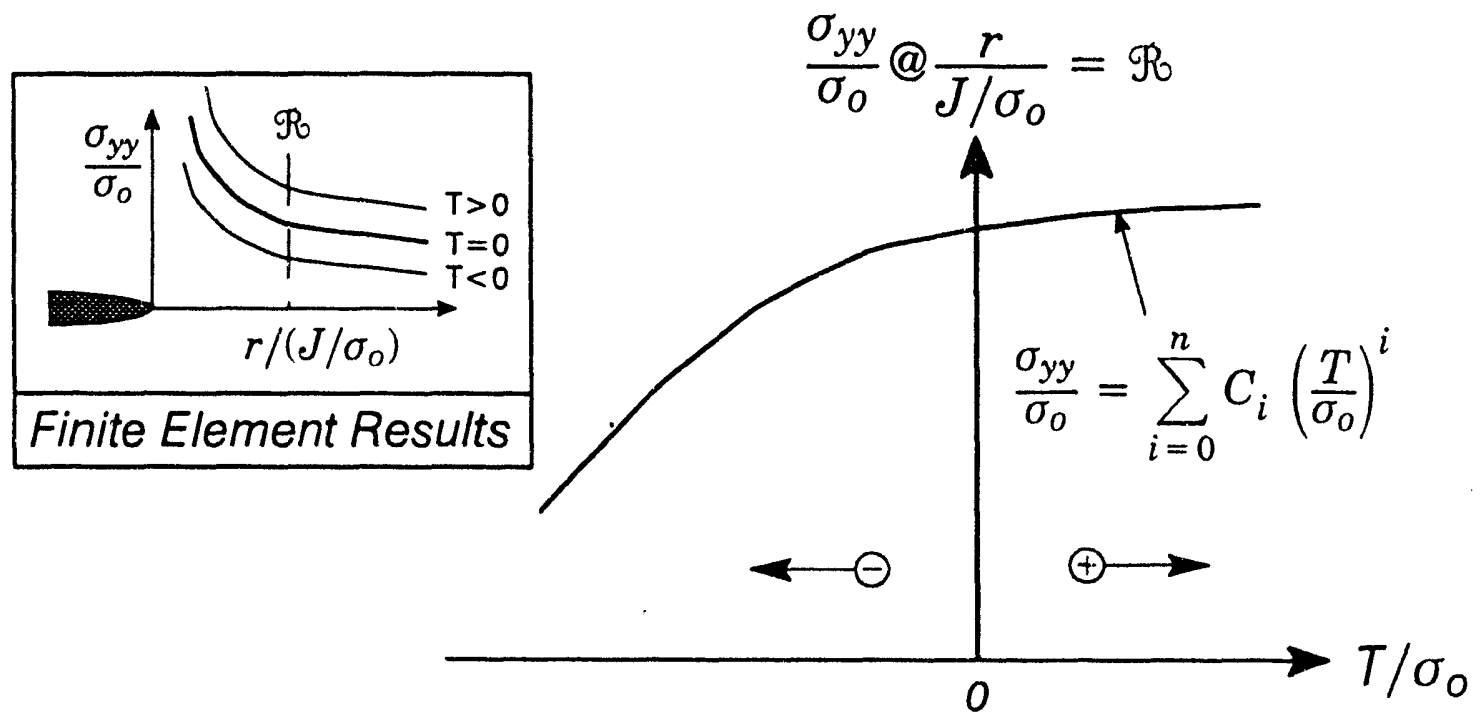

Figure 4: Relationship between opening mode stress and elastic $T$-stress quantified by a modified boundary layer model. Similar curves can be constructed at any constant normalized distance, $R$, ahead of the crack tip from finite element results. 


\section{MICROMECHANICS PREDICTIONS OF SIZE EFFECTS ON $J_{c}$}

For steels operating at temperatures where cleavage occurs after significant plastic deformation but before the initiation of ductile growth (lower to mid-transition), attainment of a critical stress over a microstructurally relevant volume is an appropriate micro-mechanical failure criteria [Ritchie, et al., 1973]. Important classes of engineering structures can fail by this mechanism, including high strength rails, offshore oil platforms, ships, pipelines storage tanks, and nuclear pressure vessels after years of neutron irradiation embrittlement. Dodds and Anderson [Dodds, et al., 1991; Anderson and Dodds, 1991, Kirk and Dodds, 1992] show that, by quantifying the effects of finite size on micro-scale / macro-scale crack driving force relations, the apparent effect of size on fracture toughness can be rigorously predicted without resort to empirical arguments. These size effects become steadily more pronounced as load increases due to the deviation of crack-tip region deformations from the $S S Y(T=0)$ conditions essential for SPFM to apply. Once SPFM becomes invalid, a micro-mechanics failure criteria is required to establish the geometry invariant conditions at fracture. Finite element analysis provides a means to quantify the geometry dependent relations between these conditions and macro-scale crack driving force. This permits (in principle) prediction of fracture in any body from toughness values measured using standard specimens.

Although cleavage is driven by stress and stressed volume, the difficulty of measuring critical values of these parameters dictates that fracture driving force, and thereby critical fracture conditions, be expressed in terms of more easily measured macroscopic parameters (e.g. $J)$. Thus, an effective macroscopic driving force for cleavage fracture $\left(J_{o}\right)$ can be defined as follows:

$J_{o}$ is the $J$ to which the infinite body ( $T=0, M B L$ model) must be loaded to achieve the same stressed volume, and thereby the same driving force for cleavage fracture, as in a finite body.

Early papers on this topic employed the notation $J_{S S Y}$ instead of $J_{o}$. The notation is changed here to emphasize that the infinite body reference configuration is $T=0$.

The variation of $J_{o}$ with $J$ is depicted schematically for two finite bodies in Figure 5 . Upon initial loading of a finite body, crack-tip plasticity is well contained within a surrounding elastic field. Crack-tip conditions are well approximated by $T=0$ and, up to some geometry dependent deformation level, $J_{o} \approx J_{\text {Finite Body. }}$. Subsequent interaction of plasticity at the crack tip with plasticity resulting from overall deformation of the structure relaxes the kinematic constraint against plastic flow at the crack tip, thus reducing the stresses in the crack-tip region below what they are for $T=0$ at the same $J$. This reduces the micro-scale driving force for cleavage. Consequently, the finite body requires more applied $J$ to achieve the same conditions for cleavage (same stressed volume) as in the infinite body. This finite size effect on crack-tip stress fields differs for different geometries constructed from the same material; it is indicated by deviation from the 1:1 slope in Figure 5. Information of this type is useful for both analysis of fracture test data and for assessing the defect integrity of structures. Path A-B-C on Figure 5 illustrates the procedure to remove geometric dependencies from experimental cleavage fracture toughness $\left(J_{c}\right)$ data by determining the geometry independent cleavage fracture toughness $\left(J_{o}\right)$ corres ponding to a measured $J_{c}$ value. Alternatively, Figure 5 permits determination of the effective driving force for cleavage fracture produced by structural loading to a certain $J_{\text {Applied }}$ value (path $\mathrm{E}-\mathrm{D}-\mathrm{C}$ ). 


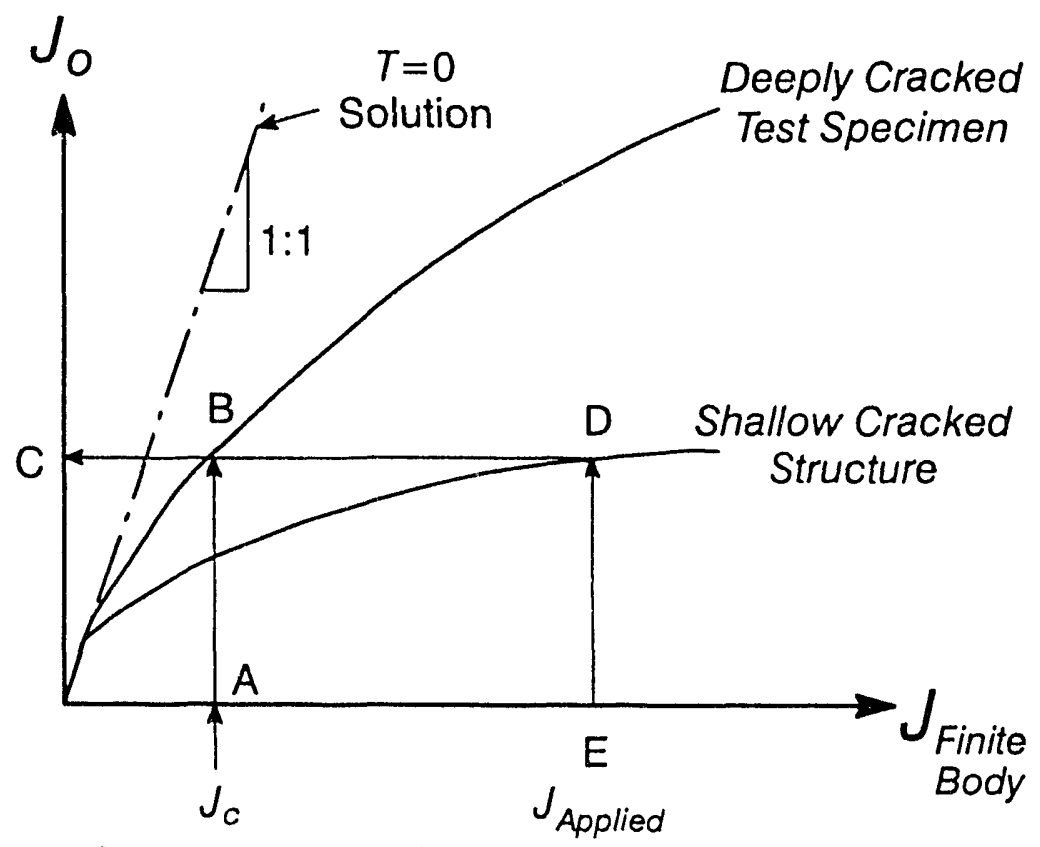

Figure 5: Conceptual variation of $J_{0}$ with $J$ for two finite bodies. Horizontal lines $\left(J_{0}=\right.$ constant) denote equivalent driving force for cleavage fracture (equivalent stressed volumes) in both bodies.

$J_{o}$ is estimated from opening mode stresses acting on the plane directly ahead of the crack tip [Dodds, et al., 1991]. The variation of these stresses with distance from the crack tip is illustrated schematically in Figure 6. Here, finite body stresses are normalized by the stress that occurs in the $T=0, M B L$ solution at the same normalized distance ahead of the crack tip (same $\left.r /\left(J /\left(a \sigma_{o} \varepsilon_{o}\right)\right)\right)$ when loaded to the same $J$ as the finite body, i.e. $J_{o} \equiv J$. Previous studies show that finite body stresses remain self-similar to the $T=0 M B L$ solution, as indicated by the radial independence of the normalized stresses in Figure 6, to deformation levels greatly exceeding those at which SPFM breaks down [Dodds, et al., 1991]. $J_{o}$ is calculated for each line on this graph as the $J$ value required in the $T=0 M B L$ solution to achieve the same opening mode stress as in the finite body. In practice, equivalence of stresses is forced at a single location ahead of the crack tip. This corresponds to selecting the critical microstructural distance $\left(l_{o}^{*}\right)$ in the Richie-Knott-Rice model [Ritchie, et al., 1973]. However, self-similarity between the $S S Y$ and finite body stress distributions makes the specific location selected unimportant over a wide range of deformation. $J_{o}$ values are independent of the critical distance selected over a range of distances that encompasses fractographically determined $l_{o}^{*}$ values [Herrens and Read, 1988; Miglin, et al., 1990]. This ability to determine $J_{o}$ irrespective of the actual $l_{o}^{*}$ value relies on self-similar stress distributions around the crack in finite and infinite bodies.

Previous investigations [Dodds, et al., 1991; Anderson and Dodds, 1991, Kirk and Dodds, 1992] employed very detailed elastic-plastic finite element analysis to quantify the effects of finite geometry and load level on opening mode stresses. The cost and time required to implement this approach make it unattractive for routine application. Conversely, opening mode stresses determined by the $T-M B L$ approximation require only an elastic analysis of the cracked structure. However, this approximation is not exact for $L S Y$. Previous investigators report differences of $\approx 10 \%$ between the $T-M B L$ approximation and opening mode stresses from full finite body calculations. These small errors are misleading due to the considerable 
influence they exert on $J_{0}$. The effect of stress errors on $J_{o}$ is quantified by assuming that the stress distribution ahead of the crack tip in a finite body is self-similar to that characteristic of $T=0$ over some range of loading, an assumption justified by reference [Dodds, et al., 1991]. Kirk and Dodds [1992] report $T=0$ solutions for a wide range of strain hardening coefficients, some of which are reproduced in Figure 7. The effect of errors in the opening mode stress at cleavage fracture on $J_{o}$ can be calculated from these results using the following equation:

$$
\frac{\left.J_{0}\right|_{T-M B L}}{J_{0}}=\frac{\bar{r}\left(\frac{o_{f}^{*}}{\sigma_{0}}, n\right)}{\bar{r}_{T-M B L}\left(\frac{o_{f}^{*}}{\sigma_{0}}(1-\xi), n\right)}
$$

where

$$
\begin{array}{ll}
\bar{r} & r /\left(J /\left(\sigma_{o} \varepsilon_{o} \alpha\right)\right) \\
\sigma_{f}^{*} & \text { cleavage fracture stress } \\
\xi & \text { percentage by which opening mode stress estimated by eqn. (8) } \\
& \text { and actual opening mode stress differ }
\end{array}
$$

Figure 8 shows that small errors in the estimated opening mode stress have proportionately greater effects on $J_{0}$, particularly for materials that do not strain harden appreciably. This example demonstrates that the seemingly small stress errors typically attributed to $T-M B L$ approximations produce unacceptably large variations in $J_{o}$. As toughness, not stress, is the quantity used when assessing structural fracture integrity, the ability of the $T-M B L$ approximation to correctly predict the effect of constraint on the effective driving force for cleavage fracture $\left(J_{o}\right)$ is quantified in this study.

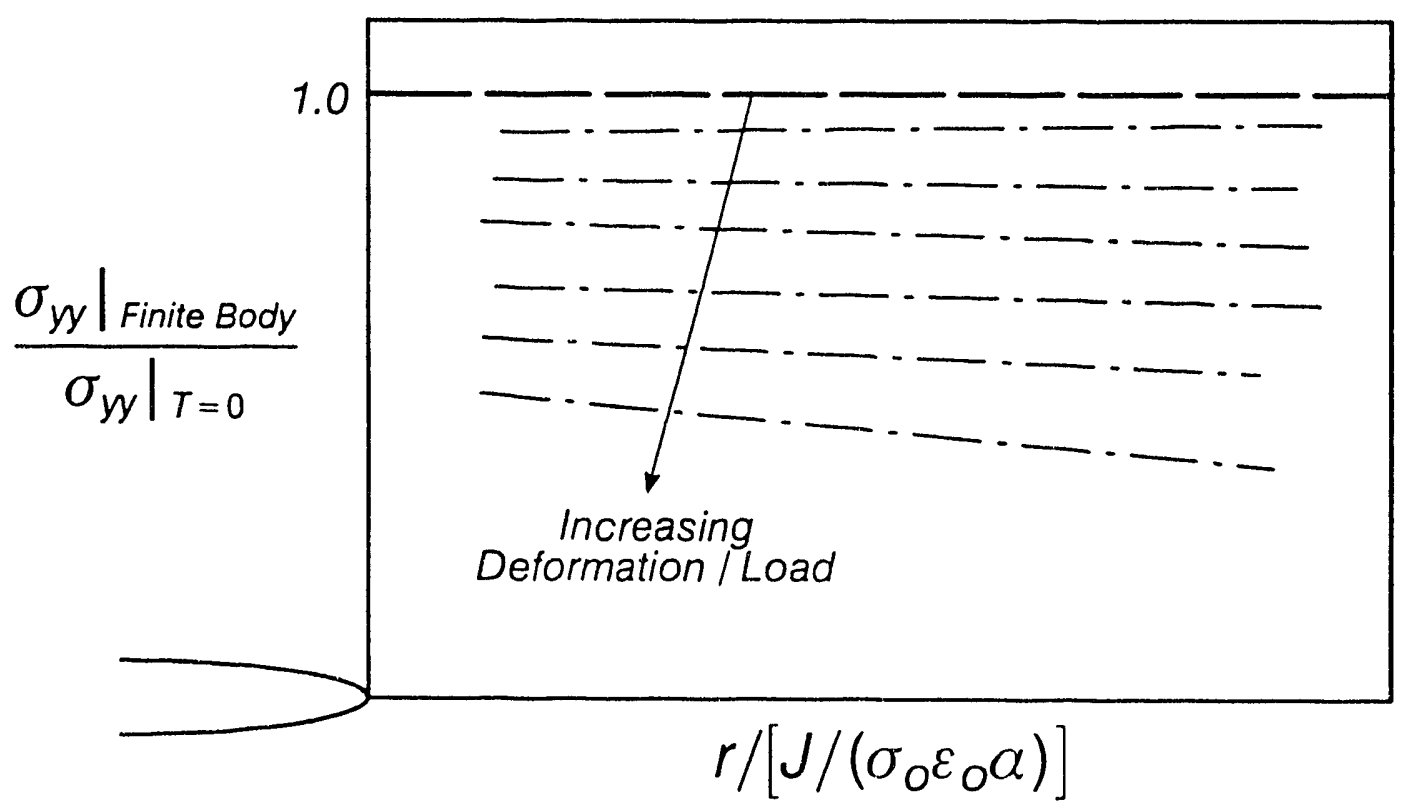

Figure 6: Effect of applied load on opening mode stresses ahead of a crack in a finite body, after [Kirk and Dodds, 1992]. 


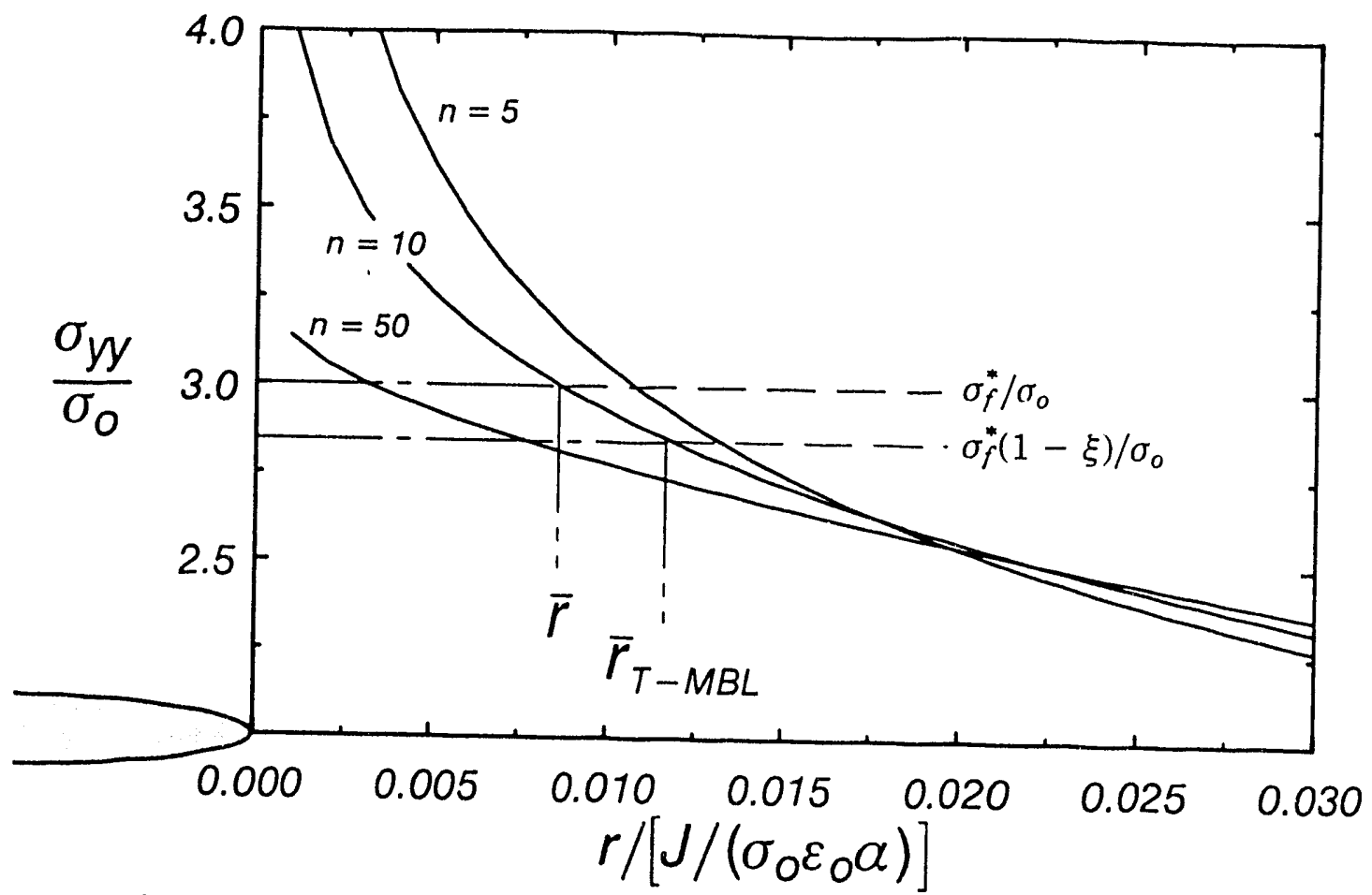

Figure 7: Opening mode stresses on the crack plane in $S S Y$ for Ramberg-Osgood materials [Kirk and Dodds, 1992].

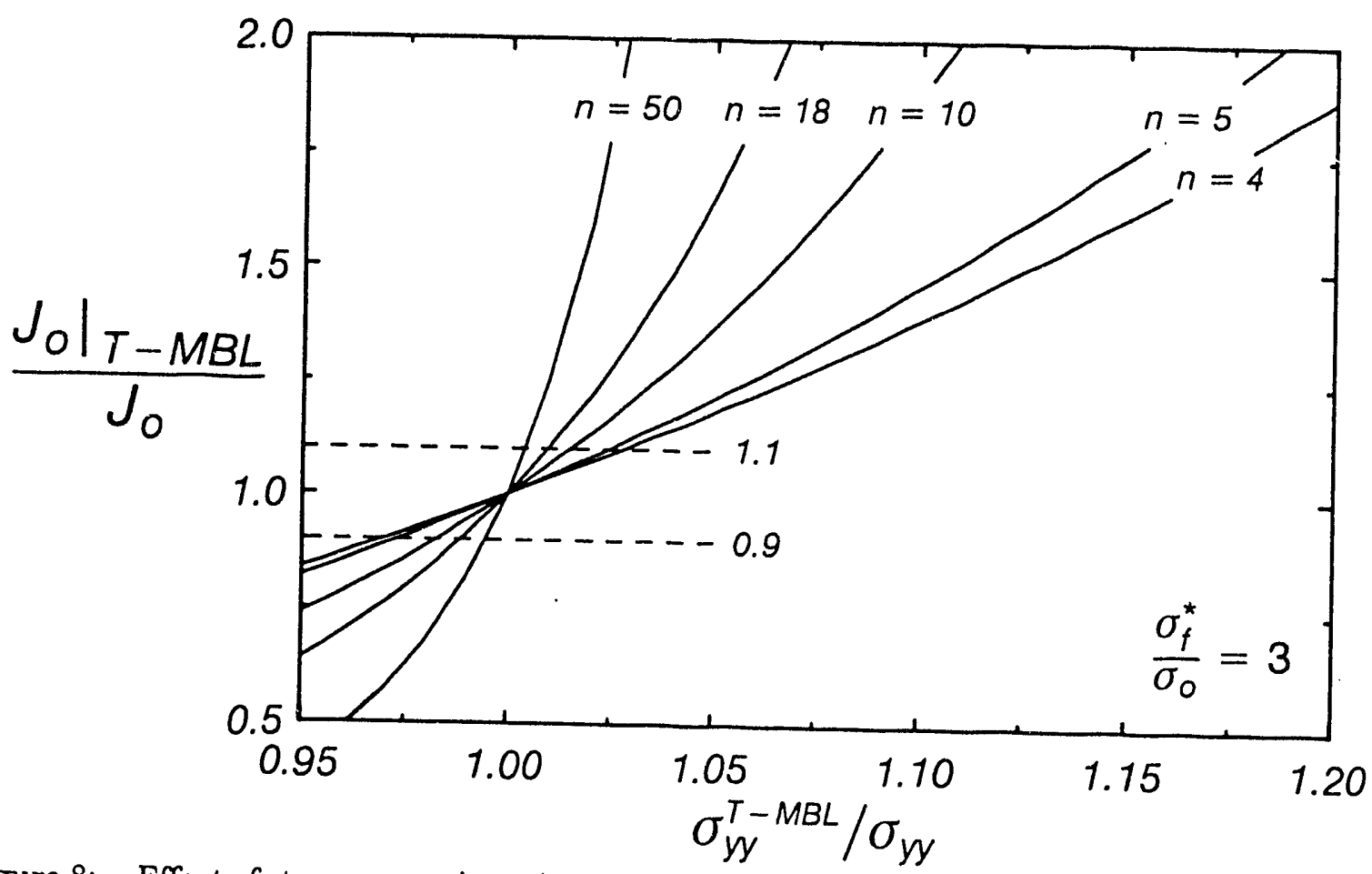

Figure 8: Effect of stress errors in estimated opening mode stress on predicted toughness values. These results depend on $\sigma_{f}^{*}$, however the relative effect of strain hardening $(n)$ is inde-
pendent of $\sigma_{f}^{*}$. 


\section{NUMERICAL PROCEDURES}

Two-dimensional, plane-strain finite element analyses of the $M B L$ model and of numerous finite cracked geometries are performed to obtain detailed resolution of stresses in the cracktip region and to determine how they change with loading. Table 1 lists the planar geometries studied along with their $\beta$ values, which range from -0.99 (very low constraint) to +2.96 (very high constraint). These analyses permit assessment of the range of conditions over which the $T-M B L$ approximation provides $J_{o}$ estimates of acceptable accuracy. Conventional small strain theory is employed throughout. These analyses are conducted using the POLO-FINITE analysis software [Dodds and Lopez, 1985] on an engineering workstation.

\subsection{Constitutive Model}

Uniaxial stress-strain behavior is described using a linear / power law model (see Figure 9) proposed by Wang [1991(a), 1991(b)]:

$$
\begin{array}{lll}
\text { Linear Region: } & \frac{\varepsilon}{\varepsilon_{o}}=\frac{\sigma}{\sigma_{o}} & \text { for } \frac{\sigma}{\sigma_{o}}<L_{1} \\
\text { Transition Region: } & \frac{\varepsilon}{\varepsilon_{o}}=\varepsilon_{N c}-\sqrt{r_{N c}^{2}-\left(\frac{\sigma}{\sigma_{o}}-\sigma_{N c}\right)^{2}} & \text { for } L_{1} \leq \frac{\sigma}{\sigma_{o}} \leq L_{2} \\
\text { Power-Law Region: } & \frac{\varepsilon}{\varepsilon_{o}}=\left(\frac{\sigma}{\sigma_{o}}\right)^{n} & \text { for } \frac{\sigma}{\sigma_{o}}>L_{2}
\end{array}
$$

where $\sigma_{o}$ is the reference stress ( $0.2 \%$ offset yield), $\varepsilon_{o}=\sigma_{o} / E$ is the reference strain, and $n$ is the strain hardening coefficient for the power-law region. The remaining parameters are defined in Figure 9. Values representing a moderately strain hardening material are used: $n=$ $10, \sigma_{\circ}=60 \mathrm{ksi}, E=30000 \mathrm{ksi}, \varepsilon_{o}=0.002$, and $L_{1}=0.95$. This constitutive model is adopted rather than the conventional Ramberg-Osgood description to ensure that SSY conditions are maintained in the $M B L$ model even for applied $T$-stresses near the yield stress. A transition region consisting of a circular arc between the linear and power-law regions makes the tangent modulus a smooth, continuous function of equivalent strain.

$J_{2}$ deformation plasticity theory (i.e. nonlinear elasticity) describes the multi-axial material behavior. By using an effective stress defined from the von Mises yield function and an effective strain defined from the Prandlt-Reuss relations, the total stress components are expressed in terms of total strain components:

$$
\frac{\sigma_{i j}}{\sigma_{o}}=\frac{1}{3(1-2 v)} \frac{\varepsilon_{k k}}{\varepsilon_{o}} \delta_{i j}+\frac{2}{3} \frac{\sigma_{e} / \sigma_{o}}{e_{e} / \varepsilon_{o}} \frac{e_{i j}}{\varepsilon_{o}}
$$

where $\varepsilon_{k k}$ is the trace of the stress tensor, $\delta_{i j}$ is the Kronecker delta, and the effective stress and strain are defined by 


$$
\begin{aligned}
& \sigma_{e}^{2}=\frac{1}{2}\left[\left(\sigma_{11}-\sigma_{22}\right)^{2}+\left(\sigma_{22}-\sigma_{33}\right)^{2}+\left(\sigma_{33}-\sigma_{11}\right)^{2}+6\left(\sigma_{12}^{2}+\sigma_{23}^{2}+\sigma_{13}^{2}\right)\right] \\
& e_{e}^{2}=\frac{2}{9}\left[\left(\varepsilon_{11}-\varepsilon_{22}\right)^{2}+\left(\varepsilon_{22}-\varepsilon_{33}\right)^{2}+\left(\varepsilon_{33}-\varepsilon_{11}\right)^{2}+\frac{3}{2}\left(\gamma_{12}^{2}+\gamma_{23}^{2}+\gamma_{13}^{2}\right)\right]
\end{aligned}
$$

\begin{tabular}{|c|c|c|c|c|c|c|c|c|c|}
\hline & $a / W$ & $H / W$ & $\begin{array}{c}\text { Loaded } \\
\text { by }\end{array}$ & $\beta$ & & $a / W$ & $H / W$ & $\begin{array}{c}\text { Loaded } \\
\text { by }\end{array}$ & $\beta$ \\
\hline \multirow{7}{*}{$\mathrm{SE}(\mathrm{B})$} & 0.05 & \multirow{7}{*}{ N/A } & \multirow{7}{*}{ Tractions } & -0.415 & \multirow{3}{*}{$\mathrm{DB}(\mathrm{T})$} & 0.25 & 0.40 & \multirow{3}{*}{ Tractions } & +1.578 \\
\hline & 0.15 & & & -0.296 & & 0.50 & 0.40 & & +2.956 \\
\hline & 0.25 & & & -0.178 & & 0.50 & 1.00 & & +0.899 \\
\hline & 0.391 & & & -0.006 & \multirow{5}{*}{$\mathrm{SE}(\mathrm{T})$} & 0.025 & 2.50 & Displ. & -0.460 \\
\hline & 0.50 & & & +0.137 & & 0.20 & 5.00 & Displ. & -0.415 \\
\hline & 0.70 & & & +0.410 & & 0.391 & 2.25 & Tractions & -0.283 \\
\hline & 0.90 & & & +1.180 & & 0.50 & 2.25 & Tractions & -0.148 \\
\hline \multirow{2}{*}{$\mathrm{DE}(\mathrm{T})$} & 0.70 & 2.25 & Tractions & -0.423 & & 0.70 & 2.25 & Tractions & +0.218 \\
\hline & 0.90 & 2.25 & Liativits & -0.273 & \multirow{2}{*}{$\mathrm{M}(\mathrm{T})$} & 0.025 & 2.50 & \multirow{2}{*}{ Displ. } & -0.994 \\
\hline \multicolumn{5}{|c|}{$\beta$ calculation by collocation } & & 0.20 & 5.00 & & -0.975 \\
\hline
\end{tabular}

\subsection{Finite Elements and Crack-Tip Modelling}

Eight noded, plane-strain isoparametric quadrilateral elements are used throughout. Reduced ( $2 \times 2$ ) Gaussian integration eliminates locking of the elemerits under incompressible plastic deformation. The crack-tip elements are collapsed into wedges with the initially coincident nodes left unconstrained to permit development of crack-tip blunting deformations. The side nodes of these elements are retained at the mid-point position. This modelling technique produces a $1 / r$ strain singularity, appropriate in the limit of perfect plasticity.

\subsection{Modified Boundary Layer (MBL) Model}

A circular domain of outer radius $R$ that contains a sharp crack tip at $r=0$ is modelled using finite elements (see Figure 1). Symmetrical boundary conditions are enforced on the crack plane. The mesh contains 3109 nodes and 986 elements divided into seventeen equally sized wedges of elements in the $\theta$ direction. Each wedge contains 58 elements whose radial dimension decreases geometrically with decreasing distance to the crack tip. Elements incident on the crack tip have a side length of $R / 530000$. Displacement increments of the 2-term elastic field, eqn. (14), are imposed on the outer circular boundary:

$$
\begin{aligned}
& \Delta \mathrm{u}(r, \theta)=\Delta K_{I} \frac{1-\nu}{E} \sqrt{\frac{r}{2 \pi}} \cos \left(\frac{\theta}{2}\right)(3-4 \nu-\cos \theta)+T \frac{1-\nu^{2}}{E} r \cos \theta \\
& \Delta \mathrm{v}(r, \theta)=\Delta K_{I} \frac{1-\nu}{E} \sqrt{\frac{r}{2 \pi}} \sin \left(\frac{\theta}{2}\right)(3-4 \nu-\cos \theta)-T \frac{v(1+\nu)}{E} r \sin \theta
\end{aligned}
$$

Displacements generating the full magnitude of the $T$-stress are applied first. Thereafter, loading occurs by displacements corresponding to increments of applied- $K$ until the plastic 
zone radius is $10 \%$ of $R$. At this point, $J$ calculated by the domain integral technique over rings embedded within the plastic zone differ from the applied $J\left(=K^{2}\left(1-v^{2}\right) / E\right)$ by less than $0.1 \%$, indicating that $S S Y$ conditions prevail. The deformation plasticity constitutive model ensures that load sequence does not influence the computed results. Application of the full $T$-stress first followed by $K$ leads to more rapid convergence of the nonlinear solution and enables verification of the self-similarity of the stresses at all $K$ levels.

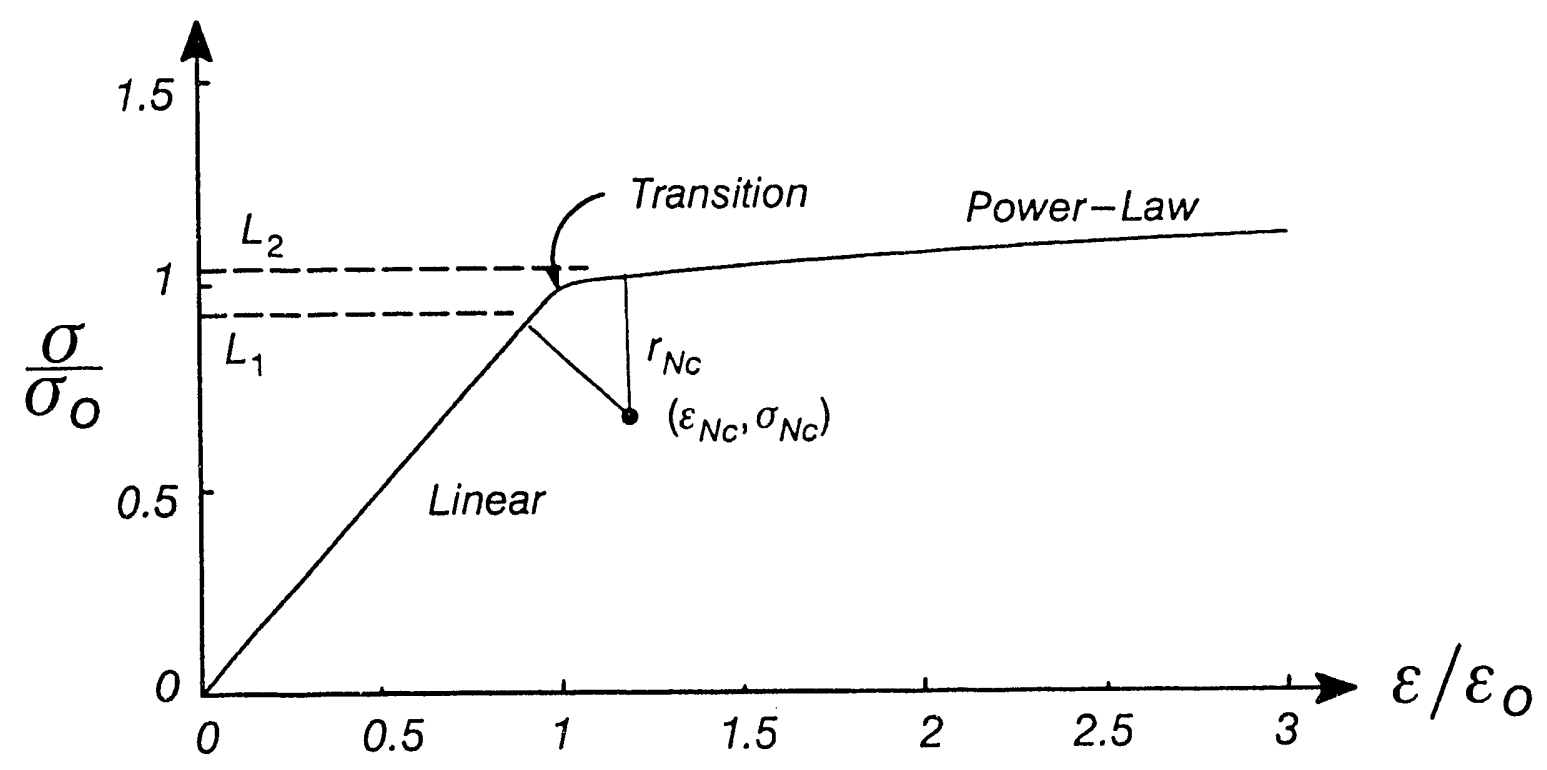

Figure 9: Uniaxial stress-strain curve defined by eqn. (10).
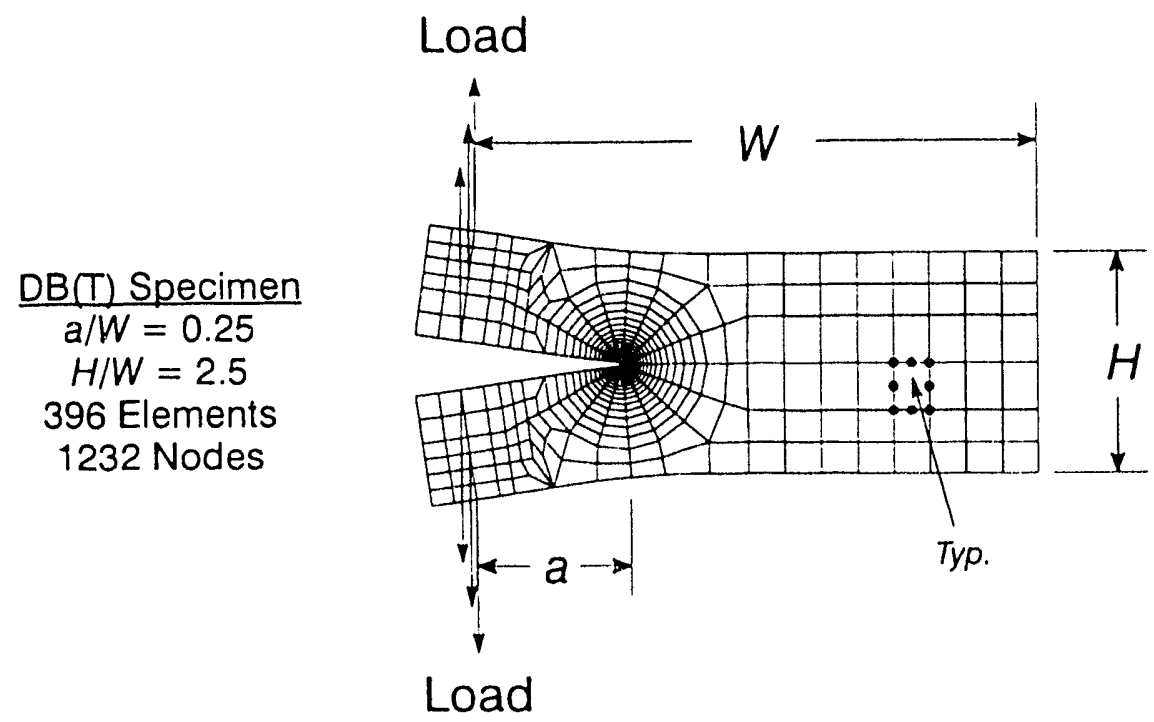

Figure 10: Finite element mesh for double cantilever beam specimen. 


\subsection{Models of Finite Geometries}

Finite element models are constructed for each cracked geometry listed in Table 1. A semi-circular core of elements surrounds the crack tip in all models. This core consists of eight equally sized wedges of elements in the $\theta$ direction. Each wedge contains 30 quadrilateral elements whose radial dimension decreases geometrically with decreasing distance to the crack tip. Crack-tip element size ranges from $0.003 \%$ to $0.22 \%$ of the crack depth depending on the crack depth modelled. All models exploit symmetry conditions where possible. Model size ranges from 343 elements / 1150 nodes for the $0.5 a / W \mathrm{DB}(\mathrm{T})$ to 1735 element $/ 5482$ nodes for the $0.2 a / W \mathrm{SE}(\mathrm{T})$ and $\mathrm{M}(\mathrm{T})$. Figure 10 illustrates a typical model.

Tension geometries are loaded by either uniform tractions or by uniform displacements applied normal to the crack plane on the remote end of the specimen. Loading by applied displacements maintains better control of the solution near the limit state for shallow cracks. $\mathrm{SE}(\mathrm{B})$ specimens are loaded by distributing uniform tractions over two small elements at the center of the compression face to eliminate the local singularity effects caused by a concentrated nodal load. $\mathrm{DB}(\mathrm{T})$ specimens are loaded by a uniform shear force applied normal to the crack plane along the centerline of the pin location in a mechanical test specimen.

\subsection{Post Processing to Obtain $J$, CTOD, $J_{o}$, and $\beta$}

The $J$-integral is computed at each load step using a domain integral method [ $\mathrm{Li}$, et al., 1985; Shih, et al., 1986]. $J$ values calculated over domains adjacent to and remote from the crack tip are within $0.003 \%$ of each other, as expected for deformation plasticity combined with these detailed meshes. CTOD is defined by the blunted shape of the crack flanks using the $\pm 45^{\circ}$ intercept procedure. $J_{0}$ is estimated by iteratively solving the following equation using a nonlinear root finder [Johnson and Riess, 1982]:

$$
\begin{aligned}
& \log \left[\frac{\left.\sigma_{y y}\right|_{F B}}{\sigma_{o}}\right]=G_{0}+G_{1} \mathbb{R}+G_{2} \mathbb{E}^{2}+G_{3} \mathbb{R}^{3}+G_{4} \mathbb{R}^{4} \text { for } 0.00085<\mathbb{R}<0.1073 \\
& \text { where, } \\
& \left.\sigma_{y y}\right|_{F B} \quad \text { finite body stress at } r=2 J_{\text {Applied }} / \sigma_{o} \\
& \text { R } \quad \log \left[r_{\text {crit }} /\left(J_{0} /\left(\sigma_{o} \varepsilon_{o}\right)\right)\right] \\
& r_{\text {crit }} \quad 2 J_{\text {Applied }} / \sigma_{o} \\
& J_{\text {Applied }} \quad J \text { applied to the finite body as computed by the domain integral } \\
& \begin{array}{lllll}
G_{0} & -1.03137 & G_{3} & -0.33021
\end{array} \\
& \begin{array}{llll}
G_{1} & -2.14688 & G_{4} & -0.03401
\end{array} \\
& G_{2} \quad-1.21929
\end{aligned}
$$

This functional form is adopted solein for convenience, i.e. a simple closed-form fit to the $T=0$ $M B L$ solution. The fit applies to a linear power law material, eqn. (10), with a strain hardening coefficient of 10 .

The biaxiality parameter $\beta$ is determined by using nodal displacements from a linear analysis of each model as input to a least-squares solution for the first twelve terms of a Westergaard series expansion. Barker employed the least-squares procedure to extract the stress 
intensity factor from experimental Morié or speckle interferometry data [Barker, et al., 1983]. For computation of $\beta$, nodal displacements are extracted from an annular region surrounding (but excluding) the crack tip having an outer radius $\leq 0.9 a$. $\beta$ is calculated by eqn. (6) as the normalized ratio of the first two series coefficients $(K$ and $T$ ). Generally estimates of $\beta$ stabilize once the number of nodal displacement conditions exceeds 40 times the number of terms in the series. $\beta$ values calculated by this technique compare favorably with those reported elsewhere [Leevers and Radon, 1982, Kfouri, 1986; Sham, 1991]. All $\beta$ values reported in Table 1 are calculated in this manner.

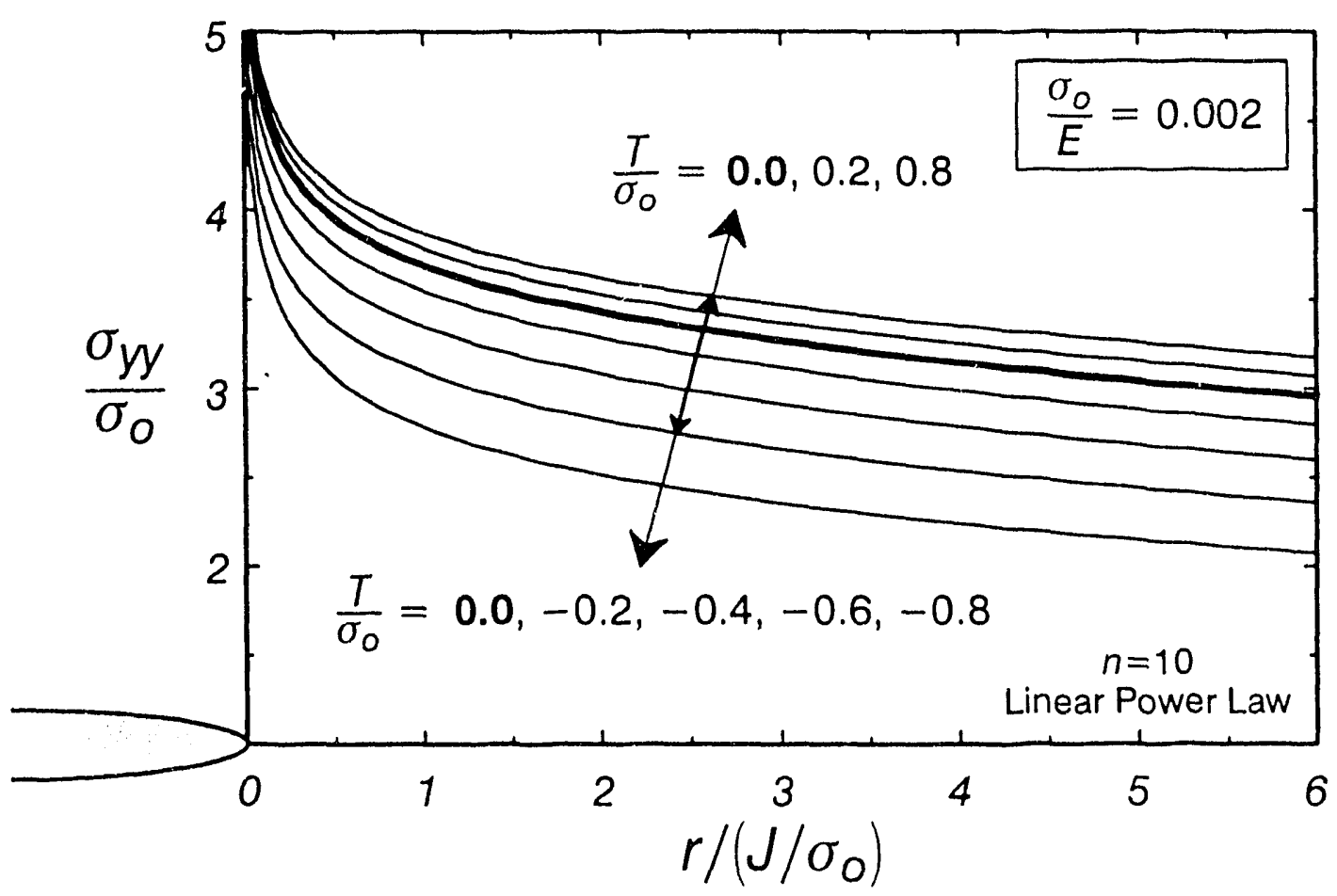

Figure 11: Opening mode stresses on the crack plane from modified boundary layer analysis 


\section{RESULTS AND DISCUSSION}

\subsection{Modified Boundary Layer Solutions}

Figure 11 shows the variation of opening mode stress in the $M B L$ solution with distance ahead of the crack tip for different applied $T$-stresses. Negative $T$ values decrease significantly the opening mode stresses below those for $T=0$ while positive $T$ values elevate slightly opening mode stresses. Approximations for crack plane stresses in finite bodies are constructed from these stress distributions by taking vertical cuts at different $r /\left(J / \sigma_{o}\right)$ values to obtain a relation between elastic $T$-stress and opening mode stress (Figure 12). The curve fit to these results for $r /\left(J / \sigma_{o}\right)=2$ facilitates comparison of this $T-M B L$ approximation to finite body stresses. Comparison at other normalized distances produces similar conclusions.

\subsection{Opening Mode Stresses in Finite Bodies}

Figure 13 compares opening mode stresses in various finite bodies to those predicted by the $T-M B L$ approximation at $r /\left(J / \sigma_{o}\right)=2$. On these figures, the zero-load condition for all finite body results occurs at $T=0, \sigma_{y y} / \sigma_{o}=3.43$ (i.e. the infinite body result). As the applied load increases, the symbols representing each finite geometry depart from this initial point. Mesh refinement limits the ability to accurately resolve near-tip stresses below a certain load, thus some geometries have no results near this initial point. The $T-M B L$ approximation accounts for only those finite geometry effects contained in the second term $(T)$ of the series expansion for stresses near the crack tip in a linear elastic body. Under SSY this is the only geometry effect so the $T-M B L$ approximation is always accurate over some initial loading rang 2 . As load increases, plastic-flow breaks to a free surface in some geometries. Un-contained yielding, not accounted for by the $T-M B L$ approximation, relieves stresses at the crack tip causing them to drop below the $M B L$ solution. On Figure 13, finite body results are plotted until the applied deformation becomes so extensive that the stress distribution loses self-similarity with the reference $T=0$ distribution. This loss of self-similarity causes ambiguity in computed $J_{o}$ values, indicating that toughness values can no longer be scaled between geometries without regard of the location of cleavage fracture initiation. An operational definition for the breakdown of self-similarity is made as the deformation beyond which $J_{o}$ values calculated at $r /\left(J / \sigma_{o}\right)=$ 1.5 and at $r /\left(\mathrm{J} / \sigma_{o}\right)=4$ differ by more than $\pm 10 \%$ of their average value.

The results in Figure 13 show that the $T-M B L$ approximation generally differs by less than $10 \%$ from the results of a full elastic-plastic analysis of the finite body. However, in certain geometries differences between the $T-M B L$ approximation and finite body results due to $L S Y$ do not appear significant until near or after self-similarity breaks down. The $T-M B L$ approximation for these specimens is much more accurate than it is for the totality of cases considered. Figure 14 re-groups the data presented in Figure 13 by $\beta$ value rather than by specimen type. Three categories emerge:

1. $|\beta|>0.9 \quad T-M B L$ stress approximation within $1.5 \%$ of finite body results

2. $|\beta|>0.4 T-M B L$ stress approximation within $3.0 \%$ of finite body results

3. $|\beta|<0.4 \quad T-M B L$ stress approximation within $17.5 \%$ of finite body results

Errors associated with very deep cracks $(a / W>0.7)$ are ignored in establishing these limits. These cases are unimportant in engineering practice. While Category 3 errors do not seem un- 


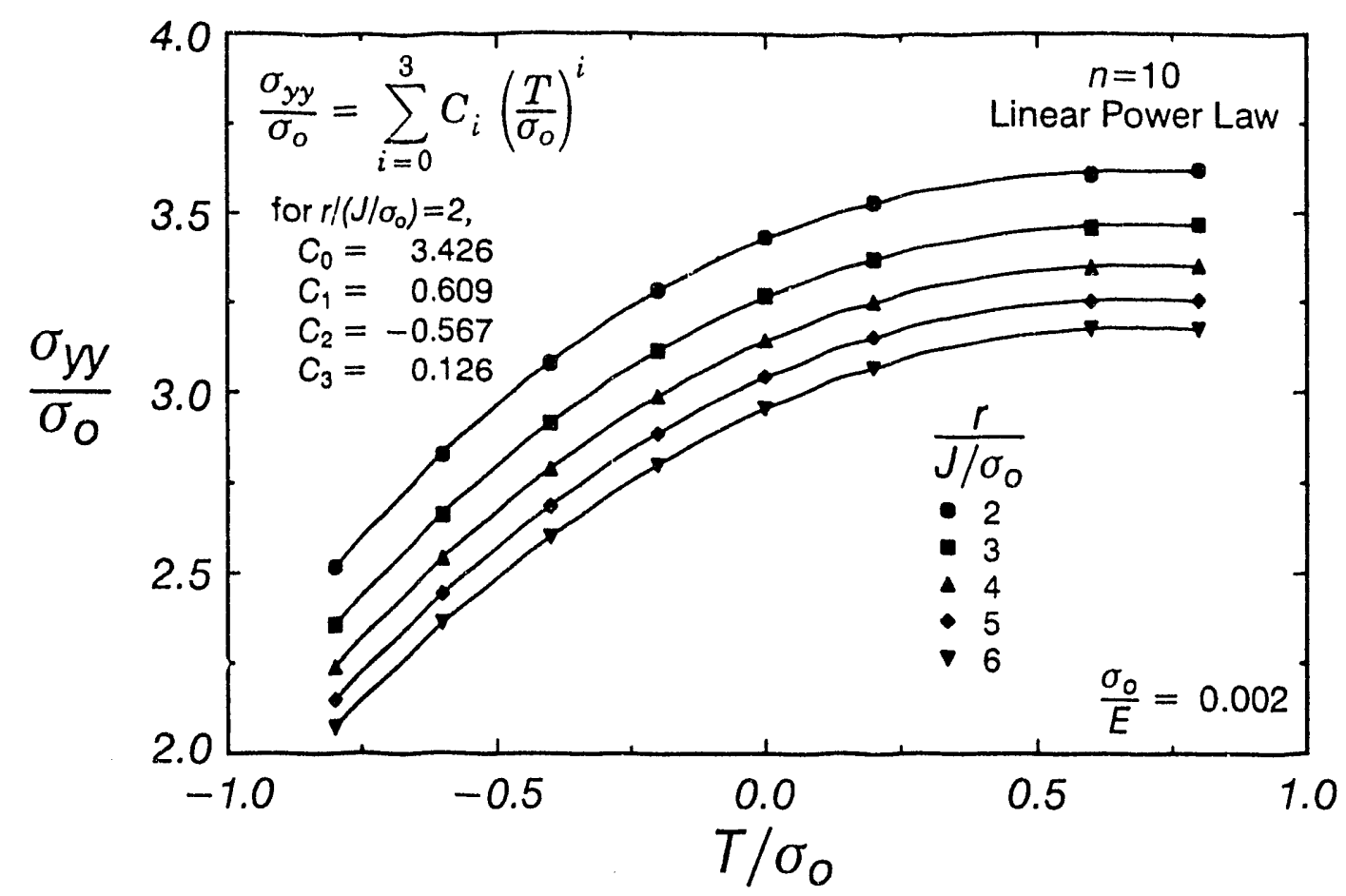

Figure 12: Variation of opening mode stress on the crack plane with applied $T$-stress and distance ahead of the crack tip determined by modified boundary layer analysis.

reasonably large, the effects of small stress errors become magnified when calculating $J_{o}$. It is encouraging that "structural type" configurations, i.e. shallow cracks in tension, fall mostly in Category 1 and 2. This suggests the possibility of estimating, with acceptable accuracy, the effective driving force for cleavage fracture, $J_{o}$, based only on elastic analysis. However, "test specimen" configurations fall mostly in Category 3 where the $T-M B L$ approximation displays limited accuracy. Fortunately, many detailed analyses of test specimen geometries have been reported in the literature, making the availability of accurate approximate techniques less important.

\section{$5.3 J_{o}$ Estimation}

Figure 15 shows the variation of $J_{o}$ with $J$ estimated by the $T-M B L$ approximation for one geometry in each Category established in Section 5.2. The reference curves shown on each figure are determined by elastic-plastic finite element analysis. Because $J$ is proportional to crack plane stresses raised to a power, the $T-M B L$ estimates are quite accurate if stress errors are small $(|\beta|>0.9)$. However, the stress errors for $|\beta|<0.4$ produce large errors in the predicted $J_{0}$. These effects are illustrated in Figure 16. Use of $T-M B L$ estimates of stress to calculate $J_{o}$ by eqn. (15) magnifies stress errors by seven to twelve fold. These results permit development of limits that must be imposed on $T-M B L$ errors in stress to keep estimates of $J_{o}$ sufficiently accurate. For example, stress errors must be below $1.5 \%$ to insure $J_{o}$ values accurate to within $\pm 10 \%$. Only geometries having $|\beta|>0.9$ satisfy this requirement. Alternatively, stress errors below $3 \%$ (i.e. specimens with $|\beta|>0.4$ ) produce $J_{o}$ estimates accurate to within $\pm 20 \%$. These error limits on $J_{o}$ are defined here only to illustrate that the $T-M B L$ approximation is much more accurate for certain geometries (high $|\beta|$ ) than for others (low $|\beta|$ ). Acceptable limits on $J_{o}$ error need to take account of the relation between $J_{o}$ and $J$ for the particular 

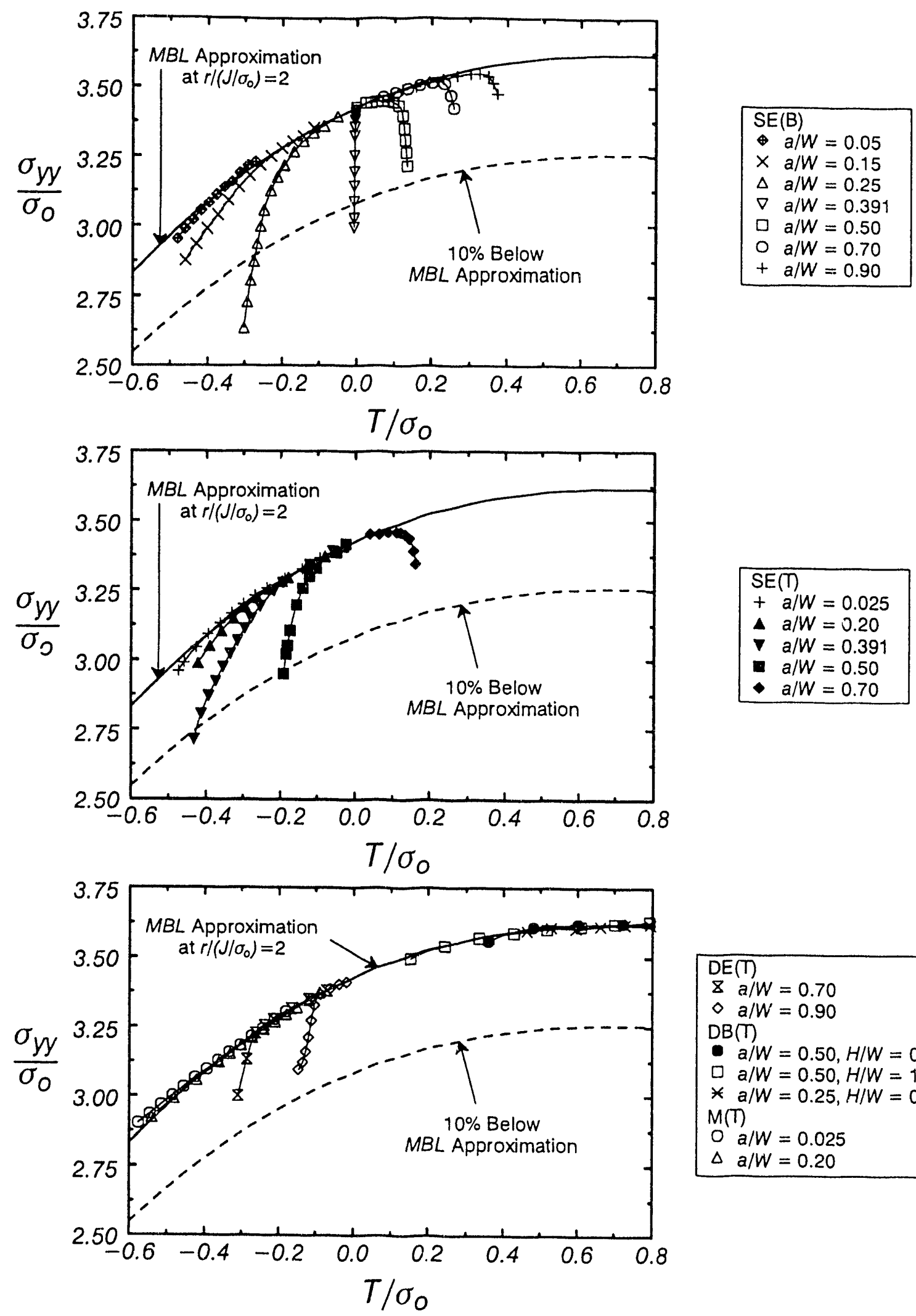

$\operatorname{DE}(T)$
$8 a / W=0.70$
$0 a / W=0.90$
$D B(T)$
$a / W=0.50, H / W=0.4$
$a / W=0.50, H / W=1.0$
$* a / W=0.25, H / W=0.4$
$M(T)$
$0 a / W=0.025$
$\Delta a / W=0.20$

Figure 13: Comparison of modified boundary layer approximation with opening mode stresses in various planar cracked geometries for an $n=10, \sigma_{o} / E=0.002$ material. 


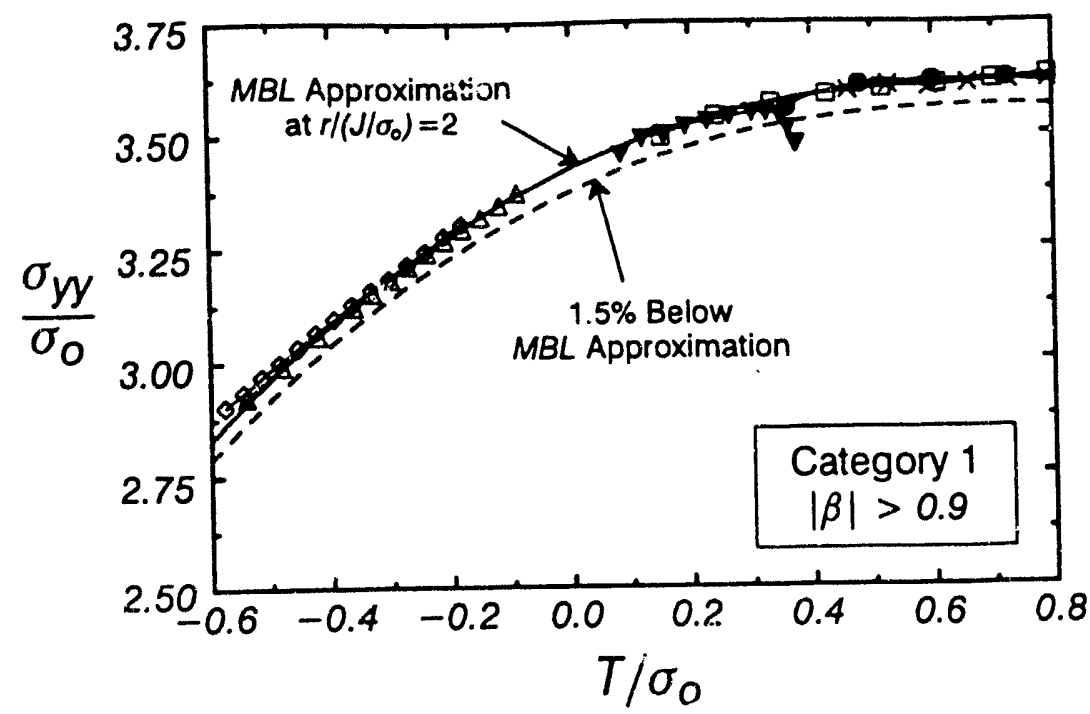

\begin{tabular}{|l|}
$S E(B)$ \\
$a / W=0.90$ \\
$D B(T)$ \\
$a / W=0.50, H / W=0.4$ \\
$a / W=0.50, H / W=1.0$ \\
$* a / W=0.25, H / W=0.4$ \\
$M(T)$ \\
$0 a / W=0.025$ \\
$\Delta a / W=0.20$
\end{tabular}

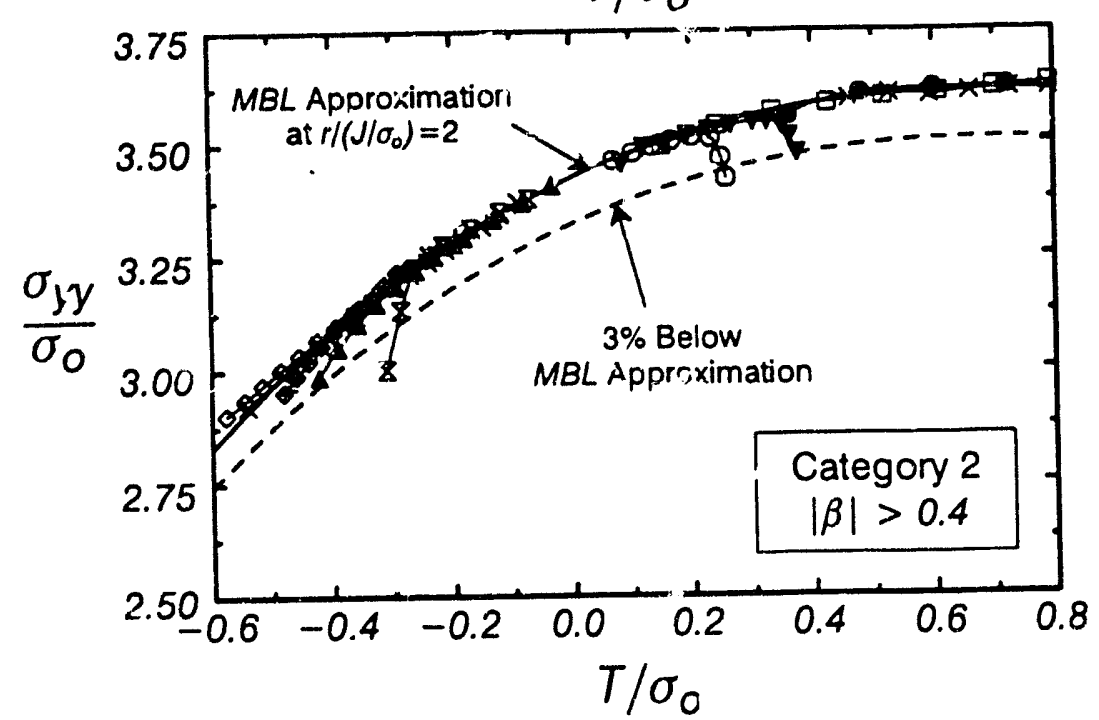

\begin{tabular}{|l}
$\mathrm{SE}(\mathrm{B})$ \\
$a / W=0.05$ \\
$0 \mathrm{a} / W=0.70$ \\
$\mathrm{a} / W=0.90$ \\
$\mathrm{SE}(\mathrm{T})$ \\
$+a / W=0.025$ \\
$\Delta a / W=0.20$ \\
$\mathrm{DE}(\mathrm{T})$ \\
$\mathrm{x} a / W=0.70$ \\
$\mathrm{DB}(\mathrm{T})$ \\
$\mathrm{a} / W=0.50, H / W=0.4$ \\
$\square \mathrm{a} / W=0.50, H / W=1.0$ \\
$* a / W=0.25, H / W=0.4$ \\
$M(T)$ \\
$0 \mathrm{a} / W=0.025$ \\
$\Delta a / W=0.20$
\end{tabular}

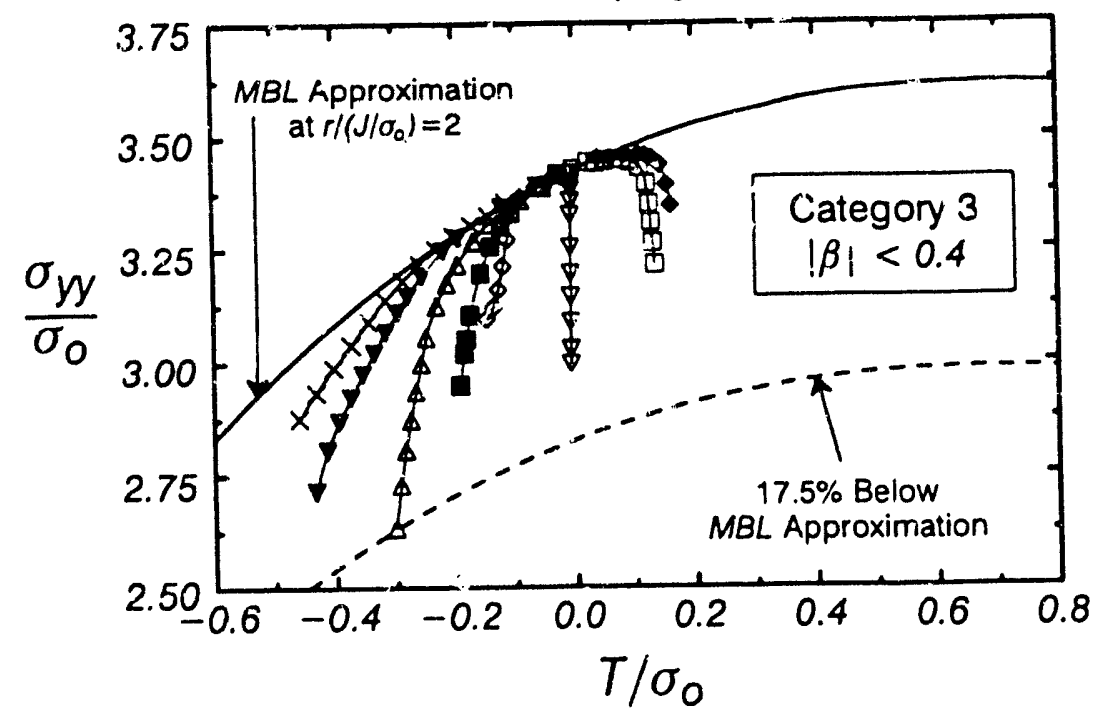

\begin{tabular}{|l|}
$\mathrm{SE}(\mathrm{B})$ \\
$\times a / W=0.15$ \\
$\Delta a / W=0.25$ \\
$\nabla a / W=0.391$ \\
$\square a / W=0.50$ \\
$\operatorname{SE}(T)$ \\
$\square a / W=0.391$ \\
$a / W=0.50$ \\
$a \mathrm{a} / W=0.70$ \\
$D E(T)$ \\
$0 \mathrm{a} / W=0.90$
\end{tabular}

Figure 14: Comparison of modif:ed boundary layer approximation with openirg mode stresses in various planar cracked geometries grouped by $|\beta|$ values for an $n=10, \sigma_{0} / E=0.002 \mathrm{ma}$. terial. 

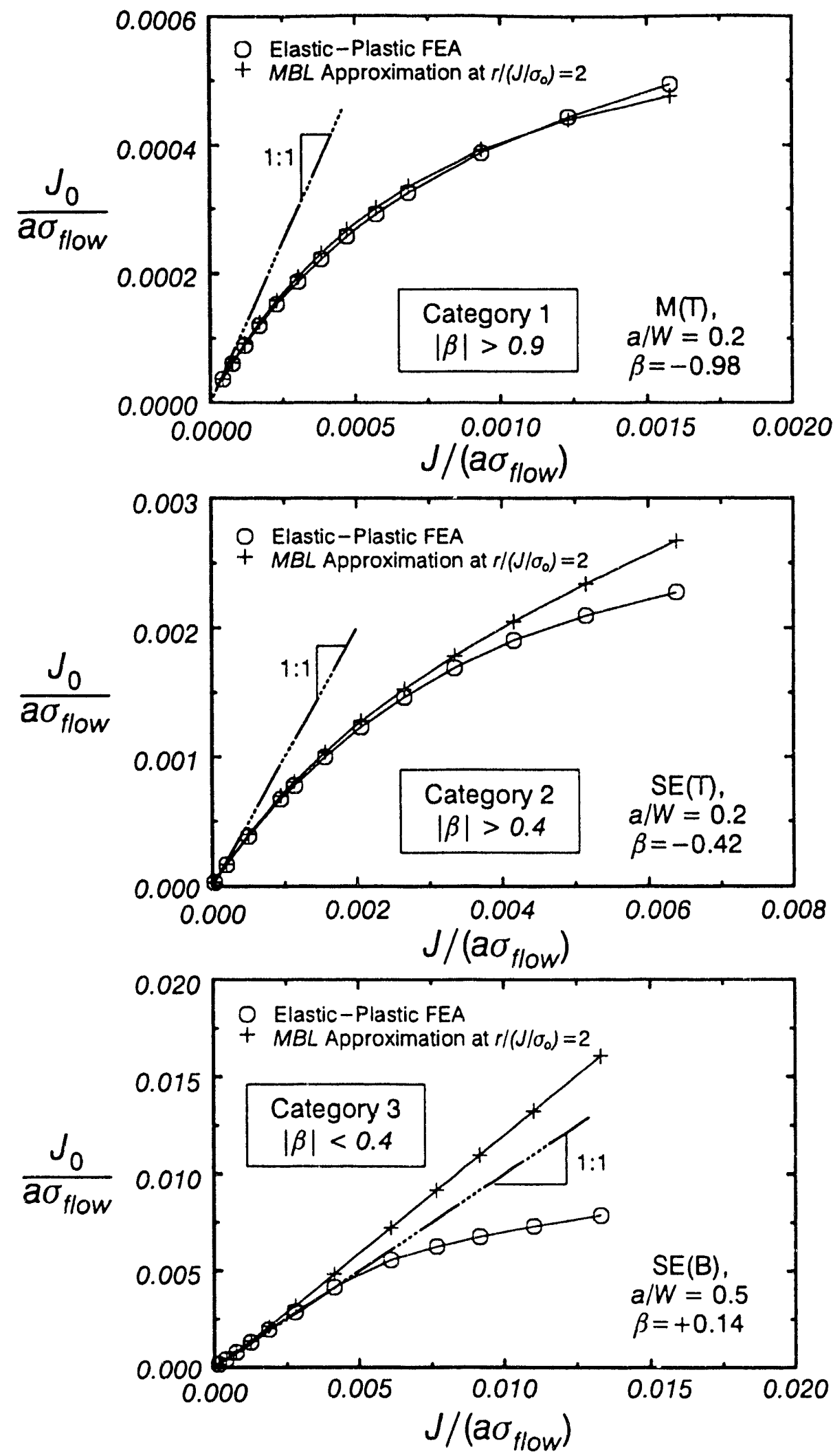

Figure 15: Comparison of constraint correction curves for cleavage fracture determined by elastic-plastic finite element analysis with those determined by the $T-M B L$ approximation for an $n=10, \sigma_{o} / E=0.002$ material. 

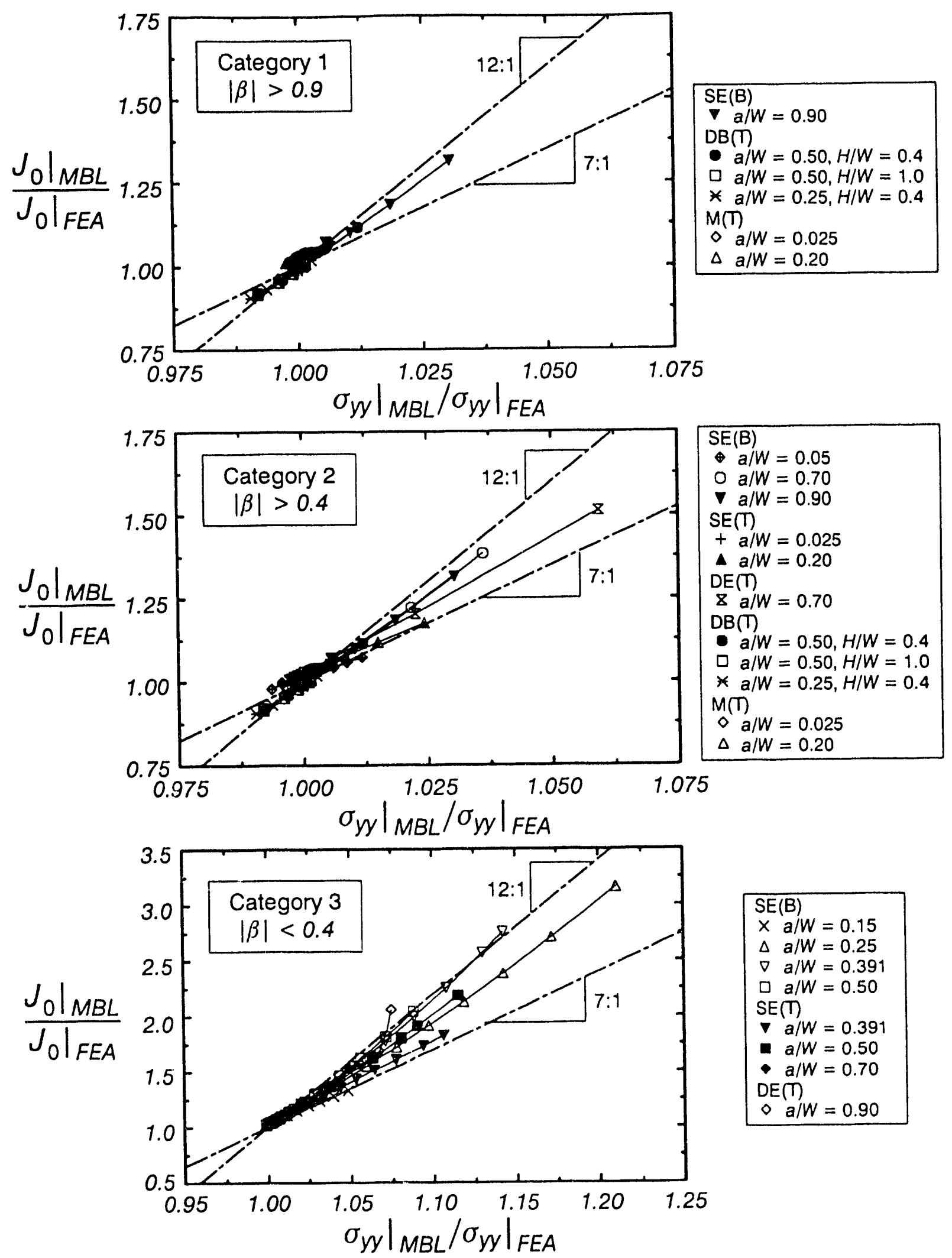

Figure 16: Effect of modified boundary layer stress estimation accuracy on $J_{o}$ estimation accuracy for an $n=10, \sigma_{o} / E=0.002$ material. 


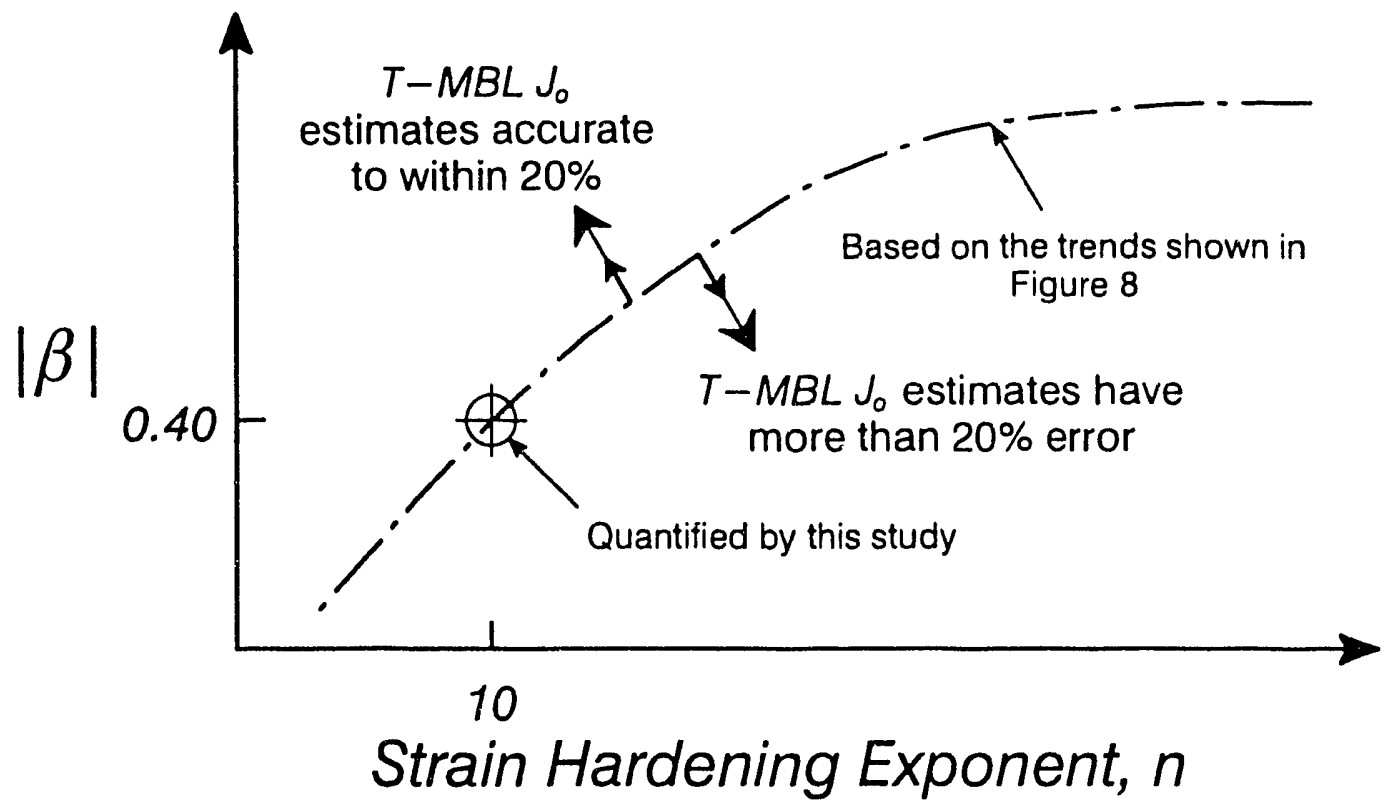

Figure 17: Postulated effect of material strain hardening on the range of biaxiality parame$\operatorname{ter}(\beta)$ over which $T-M B L J_{o}$ estimates have acceptable accuracy.

structure being assessed. As the $J_{o}$ vs. $J$ curve (Figure 15 ) becomes flat, small errors in $J_{o}$ produce much larger errors in $J$ and, consequently, in load at fracture. In this situation, tighter limits on $J_{o}$ error may be required.

These results suggest that a diagram of the type illustrated in Figure 17 can be constructed. This investigation quantifies only the point for one strain hardening exponent, $n=10$. The trend shown is conjectural based on information presented in earlier studies which suggests that increased material strain hardening (i.e. $n<10$ ) reduces the sensitivity to stress errors of the $J_{o}$ values predicted using $T-M B L$. While further analysis is needed to quantify the actual variation, it is encouraging that the low to moderate strength steels (yield stress below $580 \mathrm{MPa}$, or $84 \mathrm{ksi}$ ) used extensively in civil and offshore construction typically have strain hardening exponents below 10 [Barsom and Rolfe, 1987]. Thus, for a broad class of engineering structures, the $T-M B L$ approximation should produce acceptably accurate estimates of $J_{0}$ for a wider range of cracked geometries than reported here for $n=10$.

\subsection{Deformation Limits on the Applicability of these Fracture Analysis Methodologies}

The applicability limits of single parameter fracture mechanics (SPFM) are typically expressed as a maximum permissible deformation level relative to specimen size. So long as deformation of the structure remains below this level, the crack-tip fields in the finite body are accurately described by $J$ alone and critical $J$ values are geometry independent:

$$
D_{\text {max. }}=\frac{c}{J_{\text {crit }} / \sigma_{\text {now }}}
$$

Here $J_{c r i t}$ represents the value of $J$ at some critical event $\left(J_{c}\right.$ for cleavage fracture initiation, $J_{l c}$ for ductile fracture initiation) and $c$ is the smallest characteristic structural dimension 
(crack length, ligament length, or thickness). The smallest characteristic dimension (c) must be large compared to the CTOD (proportional to $J_{\text {crit }} / \sigma_{\text {fow }}$ ) for $J$ alone to characterize crack-tip deformations. Previous investigators have used different criteria to quantify $D_{\max }$. Shih and German [1981] establish $D_{\max }$ based on excessive deviation ( $~(10 \%)$ of crack plane stresses in finite bodies from the HRR solution. This criteria was established without regard to the fracture mechanism, although the intent was to set limits on $J_{l c}$ (ductile fracture) validity. Conversely, Dodds, et al. [1991] establish $D_{\max }$ for cleavage fracture as the deformation level above which $J_{c} / J_{o}>1$.1. This criteria requires assumption of a failure mode to establish the geometry independent conditions at fracture. While deformation limits constructed on a purely mechanical basis (e.g. deviation from HRR) are more simply determined, they are of less practical utility than micromechanically based limits, for these establish the maximum error in the quantity used to assess structural fracture integrity: toughness.

The evolving methodologies for fracture analysis discussed here extend significantly, relative to SPFM, the range of deformation over which the crack-tip stress state can be accurately described. However, these techniques also have deformation limits beyond which the cracktip fields are not uniquely and completely characterized by the parameters used. Use of any methodology beyond its applicability limit will produce apparent size dependencies in fracture toughness data. Criteria which define these limits are established as follows:

a. Limit on the T-MBL Approximation for $J_{0}: J_{0}$ cannot be estimated accurately using opening mode stresses from the $T-M B L$ approximation when $J / J_{o}$ calculated by the $T-M B L$ approximation at $r /\left(J / \sigma_{o}\right)=2$ differs by more than $10 \%$ from $J / J_{o}$ determined by elastic-plastic finite element analysis of the finite geometry.

b. Limit on $J_{0}$ from Elastic-Plastic Finite element Analysis: The effective driving force for cleavage fracture, $J_{o}$, cannot be unambiguously calculated when $J_{o}$ values calculated at $r=1.5 \mathrm{~J} / \sigma_{o}$ and at $r=1.5 \mathrm{~J} / \sigma_{o}$ differ by more than $10 \%$ of their average value.

As shown in Figure 18, it appears that $\beta$ parameterizes these deformation limits, effectively accounting for the combined effects of geometry and loading mode. While this relationship lacks a rigorous basis, it is not completely unforeseen that $\beta$ should account for geometry and loading mode effects as $\beta$ depends on both. However, as $\beta$ is only an elastic quantity, the effect of material strain hardening remains unquantified. 

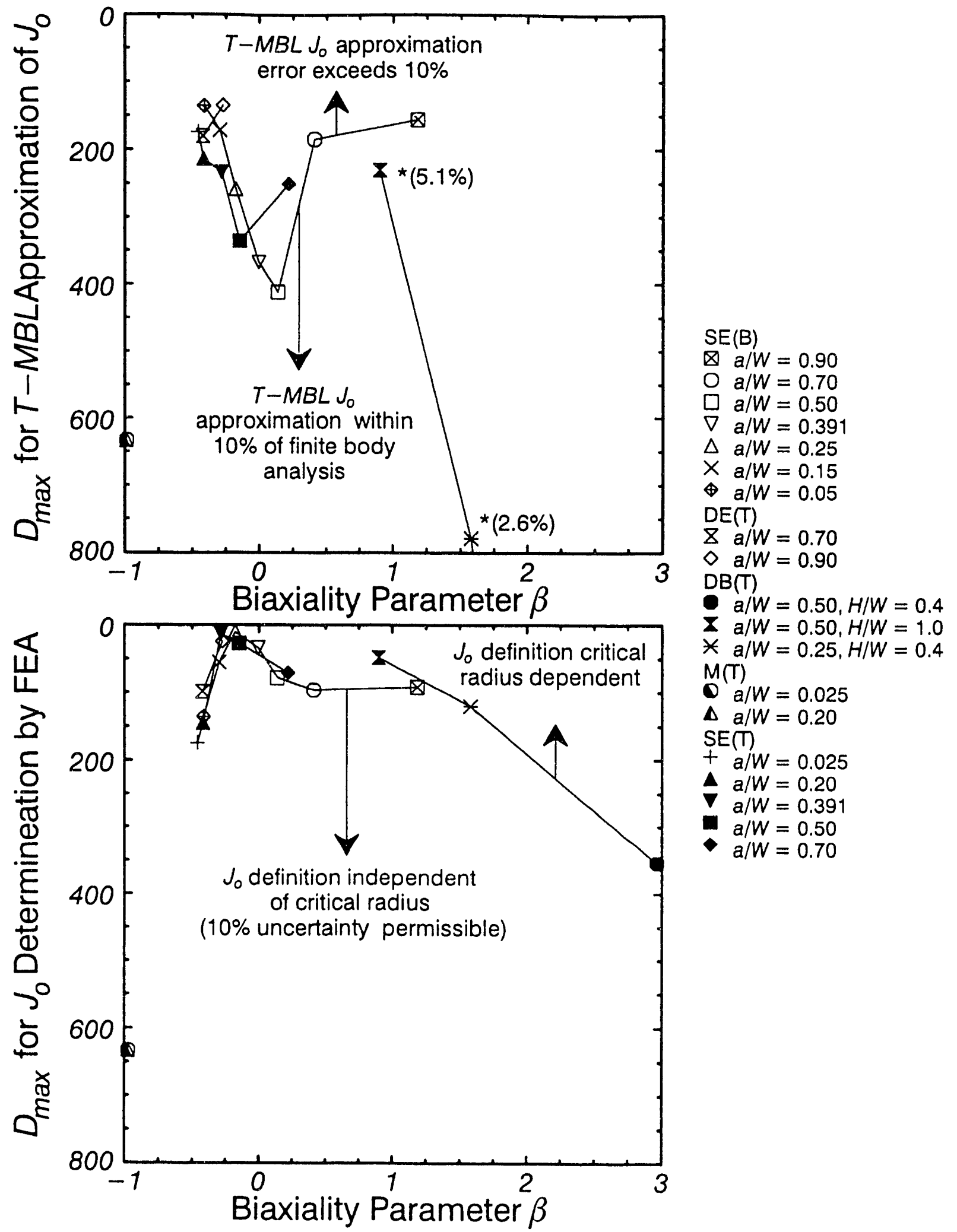

Figure 18: Deformation limits on the $T-M B L$ approximation for $J_{0}$ (top graph) and on elastic-plastic finite element calculation of $J_{o}$ (bottom graph) for an $n=10, \sigma_{o} / E=0.002$ material. Symbols representing specimens of same type are connected by lines. Symbols on top graph marked with asterisks denote deformation when $T / \sigma_{0}=0.8$. At this deformation the $T-M B L$ approximation has the error given in parenthesis. 


\section{SUMMARY AND CONCLUSIONS}

This investigation examines the ability of an elastic $T$-stress analysis coupled with a modified boundary layer $(M B L)$ solution to predict stresses ahead of a crack tip in a variety of planar geometries. The approximate stresses are used as input to estimate the effective driving force for cleavage fracture $\left(J_{o}\right)$ using the micromechanically based approached introduced by Dodds and Anderson. Finite element analyses for a wide variety of planar cracked geometries are conducted which have elastic biaxiality parameters $(\beta)$ ranging from -0.99 (very low constraint) to +2.96 (very high constraint). The magnitude and sign of $\beta$ indicate the rate at which crack-tip constraint changes with increasing applied load. All results pertain to a moderately strain hardening hardening material (strain hardening exponent $(n)$ of 10 ). The following specific conclusions may be drawn from these analyses:

1. The accuracy of the $T-M B L$ approximation for opening mode stresses strongly correlates with the elastic biaxiality parameter $\beta$ for planar geometries. The following limits are established by comparing $T-M B L$ estimates to finite body calculations:

$|\beta|>0.9 T-M B L$ stress approximation within $1 \%$ of finite body results

$0.4<|\beta|<0.9 T-M B L$ stress approximation within $2.5 \%$ of finite body results

$|\beta|<0.4 \quad T-M S L$ stress approximation within $17.5 \%$ of finite body results

2. These errors in the estimated stress become magnified by a factor of 7 to 12 when used to calculate the effective driving force for cleavage fracture $\left(J_{0}\right)$ because $J$ scales with stress raised to a power. However, for a restricted range of geometries, reasonable accuracy is available:

$|\beta|>0.9 \quad T-M B L$ approximation of $J_{S S Y}$ within $10 \%$ of finite body results

$0.4<|\beta|<0.9 T-M B L$ approximation of $J_{0}$ within $25 \%$ of finite body results

As "structural type" configurations, i.e. shallow cracks in tension, generally have $|\beta|>0.4$, it appears that only an elastic analysis is needed to determine reasonably accurate $J_{o}$ values for structural conditions.

3. $\beta$ parameterizes deformation limits on the applicability of evolving fracture methodologies ((i.e. $T-M B L, J-Q$, and $J / J_{o}$ ), effectively accounting for the combined effects of geometry and loading mode.

Available evidence suggests that increased material strain hardening (i.e. $n<10$ ) will increase the range of cracked geometries for which the $T-M B L$ approximation is sufficiently accurate. The low to moderate strength steels (yield stress below $580 \mathrm{MPa}$, or $84 \mathrm{ksi}$ ) used extensively in civil and offshore construction typically have strain hardening exponents below 10 . Thus, for a broad class of engineering structures, the $T-M B L$ approximation should produce acceptably accurate estimates of $J_{o}$ for a wider range of cracked geometries than reported here for $n=10$. 


\section{REFERENCES}

Al-Ani, A.M., and Hancock, J.W., "J-Dominance of Short Cracks in Tension and Bending," Journal of Mechanics and Physics of Solids, Vol. 39, pp. 23-43, 1991.

Anderson, T.L., and Dodds, R.H., "Specimen Size Requirements for Fracture Toughness Testing in the Ductile-Brittle Transition Region," Journal of Testing and Evaluation, Vol. 19, pp. 123-134, 1991.

Barker, D.B., Sanford, R.J., and Chona, R., "Determining $K$ and Related Stress Field Parameters from Displacement Fields," Proceedings, Society for Experimental Stress Analysis, Bethel, Pennsylvania, 1983.

Barsom, J.M., and Rolfe, S.T., Fracture and Fatigue Control in Structures - Applications of Fracture Mechanics, Prentice Hall, Inc., Englewood Cliffs, New Jersey, p. 265, 1987.

Bertegon, C., and Hancock, J.W., "Two-Parameter Characterization of Elastic-Plastic Crack Tip Fields," Journal of Applied Mechanics, Vol. 58, pp. 104-113, March 1991.

Dodds, R.H., and Lopez, L.A., "Software Virtual Machines for Development of Finite-Element Systems," International Journal for Engineering with Computers, Vol. 13, pp. 18-26, 1985.

Dodds, R.H., Anderson, T.L., and Kirk, M.T., "A Framework to Correlate $a$ / W Ratio Effects on ElasticPlastic Fracture Toughness $\left(J_{c}\right)$," International Journal of Fracture, Vol. 48, pp. 1-22, 1991.

Du, Z.Z., and Hancock, J.W., "The Effect of Non-Singular Stresses on Crack Tip Constraint," Journal of the Mechanics and Physics of Solids, Vol. 39, pp. 555-567, 1991.

Herrens, J., and Read, D.T., "Fracture Behavior of a Pressure Vessel Steel in the Ductile-to-Brittle Transition Region," NISTIR 88-3099, National Institute for Standards and Technology, Boulder, Colorado, December, 1988.

Hutchinson, J.W., "Singular Behavior at the End of a Tensile Crack in a Hardening Material," Journal of Mechanics and Physics of Solids, Vol. 16, pp. 13-31, 1968.

Johnson, L.W., and Riess, R.D., Numerical Analysis, Addison-Wesley Publishing Co., Reading, Massachusetts, p.145, 1982.

Keeney-Walker, J., Bass, B.R., and Landes, J.D., "An Investigation of Crack Tip Stress-Field Criteria for Predicting Cleavage Crack Initiation," to appear in ASTM STP 1131, 1993.

Kfouri, A.P., "Some Evaluations of the Elastic T-term using Eshelby's Method," International Journal of Fracture, Vol. 30, pp. 301-315, 1986.

Kirk, M.T., and Dodds, R.H., "An Analytical and Experimental Comparison of $J_{i}$ Values for Shallow Through and Part Through Surface Cracks," Engineering Fracture Mechanics, Vol. 39, No. 3, pp.
535-551, 1991.

Kirk, M.T., and Dodds, R.H., "The Influence of Weld Strength Mismatch on Crack-Tip Constraint in Single Edge Notch Bend Specimens," to appear in the International Journal of Fracture, 1993.

Larsson, S.G., and Carlsson, A.J., "Influence of Non-Singular Stress Terms and Specimen Geometry on Small-Scale Yielding at Crack Tips in Elastic-Plastic Materials," Journal of the Mechanics and Physics of Solids, Vol. 21, pp. 263-277, 1973.

Leevers, P.C. and Radon, J.C., "Inherent Stress Biaxiality in Various Specimen Geometries," International Journal of Fracture, Vol. 19, pp. 311-325, 1982.

Li, F.Z., Shih, C.F., and Needleman, A., "A Comparison of Methods for Calculating Energy Release Rates," Engineering Fracture Mechanics, Vol. 21, pp. 405-421, 1985.

McMeeking, R.M., "Finite Deformation Analysis of Crack-Tip Opening in Elastic-Plastic Materials and Implications for Fracture," Journal of the Mechanics and Physics of Solids, Vol. 25, pp.

Miglin, M.T., Wade, C.S., and Van Der Sluys, W.A., "Analysis of Fracture Toughness Data for Modified SA508 C12 in the Ductile-to-Brittle Transition Region, "Fracture Mechanics: Twenty-First Symposium, ASTM STP 1074, J.P. Gudas, J.A. Joyce, and E.M. Hackett, Eds., American Society for Testing and Materials, Philadelphia, Pennsylvania, pp. 238-263, 1990. 
Moran, B., and Shih, C.F., "Crack Tip and Associated Domain Integrals from Momentum and Energy Balance," Engineering Fracture Mechanics, Vol. 27, pp. 615-642, 1987.

Nakamura, T., and Parks, D., "Determination of Elastic T-Stress Along 3-D Crack Fronts Using an Elastic Interaction Integral," submitted for publication in the International Journal of Fracture, 1992.

O'Dowd, N.P., and Shih, C.F., "Family of Crack-Tip Fields Characterized by a Triaxiality Parameter: Part I - Structure of Fields," Journal of the Mechanics and Physics of Solids, Vol. 39., No. 8, pp. 989-1015, 1991.

O'Dowd, N.P., and Shih, C.F., "Family of Crack-Tip Fields Characterized by a Triaxiality Parameter: Part II - Fracture Applications," Journal of the Mechanics and Physics of Solids, Vol. 40, pp. 939-963, 1992.

Parks, D.M., "Engineering Methodologies for Assessing Crack Front Constraint," Proceedings, Spring Meeting of the Society for Experimental Mechanics, Milwaukee, Wisconsin, 1991.

Rice, J.R., and Rosengren, G.F., "Plane Strain Deformation Near a Crack Tip in a Power-Law Hardening Material," Journal of Mechanics and Physics of Solids, Vol. 16, pp. 1-12, 1968.

Rice, J.R., and Tracey, D.M., in Numerical and Computer Methods in Structural Mechanics, S.J. Fenves et al. (eds.), Academic Press, New York, pp. 585-623, 1968.

Ritchie, R.O., Knott, J.F., and Rice, J.R., "On the Relationship Between Critical Tensile Stress and Fracture Toughness in Mild Steel," Journal of the Mechanics and Physics of Solids, Vol. 21, pp. $395-410,1973$.

Sham, T.L., "The Determination of the Elastic T-term using Higher Order Weight Functions," International Journal of Fracture, Vol. 48, pp. 81-102, 1991.

Shih, C.F. and German, M.D., "Requirements for a One Parameter Characterization of Crack Tip Fields by the HRR Singularity," International Journal of Fracture, Vol. 17, No. 1, pp. 27-43, 1981.

Shih, C.F., Moran, B., and Nakamura, T., "Energy Release Rate Along a Three-Dimensional Crack Front in a Thermally Stressed Body," International Journal of Fracture, Vol. 30, pp. 79-102, 1986.

Sorem, W.A., Dodds, R.H., and Rolfe, S.T., "Effects of Crack Depth on Elastic Plastic Fracture Toughness," International Journal of Fracture, Vol. 47, pp. 105-126, 1991.

Sumpter, J.D.G., and Hancock, J.W., "Shallow Crack Toughness of HY-80 Welds: An Analysis Based on T Stresses," International Journal of Pressure Vessels and Piping, Vol. 45, pp. 207-221, 1991.

Sumpter, J.D.G., and Forbes, A.T., "Constraint Based Analysis of Shallow Cracks in Mild Steel," Proceedings of the International Conference on Shallow Crack Fracture Mechanics Tests and Applications, TWI, Cambridge, England, September 1992.

Theiss, T.J., and Bryson, J.R., "Influence of Crack Depth on Fracture Toughness of Reactor Pressure Vessel Steel," Constraint Effects in Fracture, ASTM STP 1171, E.M. Hackett, K-H. Schwalbe, and R. H. Dodds, Eds., American Society for Testing and Materials, Philadelphia, Pennsylvania, pp. 104-119, 1993.

Wang, Y.Y., "On the Two-Parameter Characterization of Elastic-Plastic Crack-Front Fields in Surface-Cracked Plates," Constraint Effects in Fracture, ASTM STP 1171, E.M. Hackett, K.-H. Schwalbe, and R. H. Dodds, Eds., American Society for Testing and Materials, Philadelphia, Pennsylvania, pp. 120-138, 1993.

Wang, Y.Y., "A Two-Parameter Characterization of Elastic-Plastic Crack Tip Fields and Applications to Cleavage Fracture, Ph.D. Dissertation, MIT, 1991(b). 


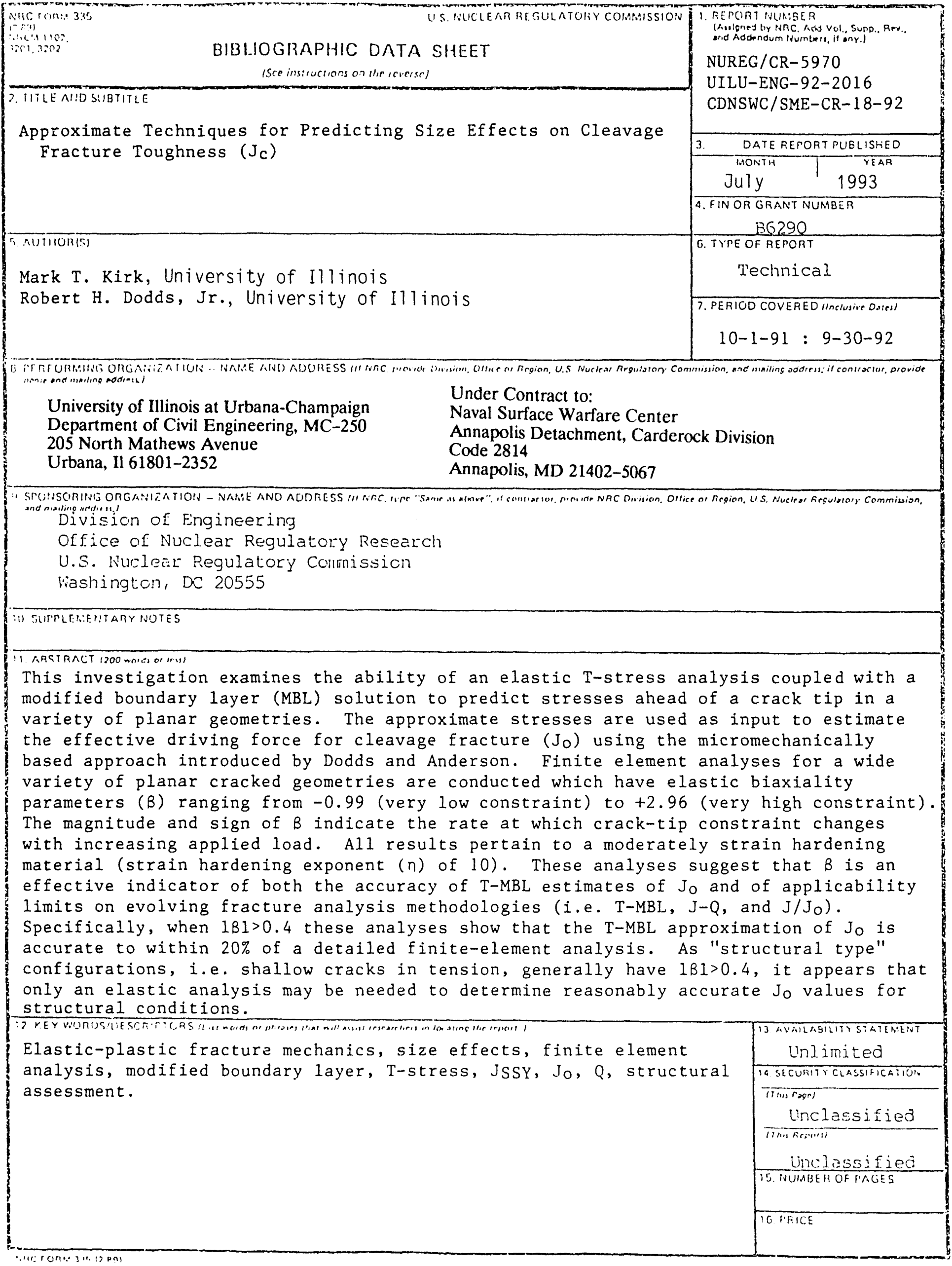



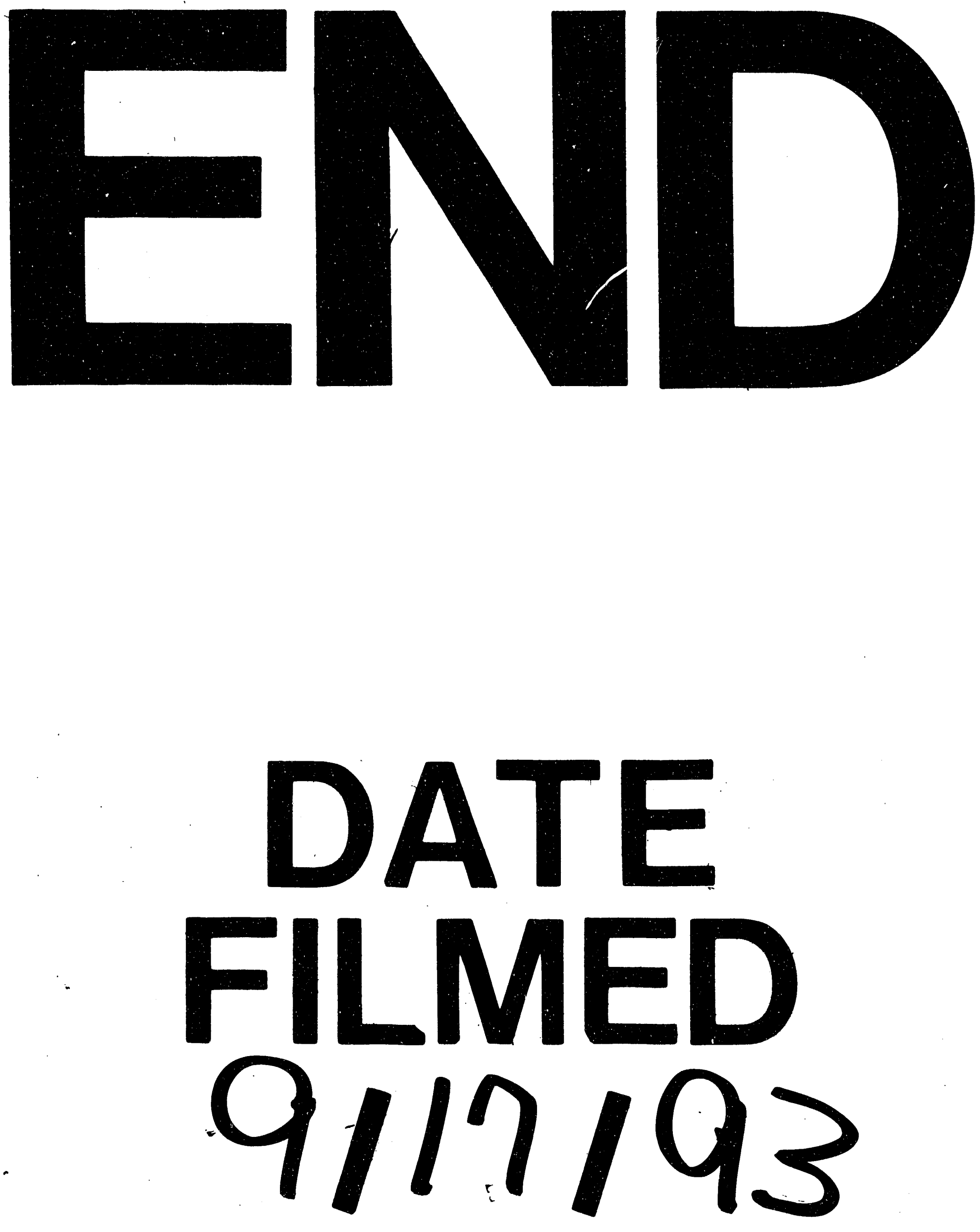
